\title{
Chromosomal microarray analysis in the genetic evaluation of 279 patients with syndromic obesity
}

Carla Sustek D'Angelo ${ }^{1 *}$, Monica Castro Varela ${ }^{1}$, Claudia Irene Emílio de Castro ${ }^{1}$, Paulo Alberto Otto', Ana Beatriz Alvarez Perez ${ }^{2}$, Charles Marques Lourenço ${ }^{3}$, Chong Ae Kim ${ }^{4}$, Debora Romeo Bertola ${ }^{4}$, Fernando Kok ${ }^{5}$, Luis Garcia-Alonso ${ }^{2}$ and Celia Priszkulnik Koiffmann ${ }^{3}$

\begin{abstract}
Background: Syndromic obesity is an umbrella term used to describe cases where obesity occurs with additional phenotypes. It often arises as part of a distinct genetic syndrome with Prader-Willi syndrome being a classical example. These rare forms of obesity provide a unique source for identifying obesity-related genetic changes. Chromosomal microarray analysis (CMA) has allowed the characterization of new genetic forms of syndromic obesity, which are due to copy number variants (CNVs); however, CMA in large cohorts requires more study. The aim of this study was to characterize the CNVs detected by CMA in 279 patients with a syndromic obesity phenotype.

Results: Pathogenic CNVs were detected in 61 patients (22\%) and, among them, 35 had overlapping/recurrent CNVs. Genomic imbalance disorders known to cause syndromic obesity were found in $8.2 \%$ of cases, most commonly deletions of 1p36, 2q37 and 17p11.2 (5.4\%), and we also detected deletions at 1p21.3, 2p25.3, 6q16, 9q34, 16p11.2 distal and proximal, as well as an unbalanced translocation resulting in duplication of the GNB3 gene responsible for a syndromic for of childhood obesity. Deletions of $9 p$ terminal and $22 q 11.2$ proximal/distal were found in $1 \%$ and $3 \%$ of cases, respectively. They thus emerge as being new putative obesity-susceptibility loci. We found additional CNVs in our study that overlapped with CNVs previously reported in cases of syndromic obesity, including a new case of 13q34 deletion (CHAMP1), bringing to 7 the number of patients in whom such defects have been described in association with obesity. Our findings implicate many genes previously associated with obesity (e.g. PTBP2, TMEM18, MYT1L, POU3F2, SIM1, SH2B1), and also identified other potentially relevant candidates including TAS1R3, ALOX5AP, and GAS6.

Conclusion: Understanding the genetics of obesity has proven difficult, and considerable insight has been obtained from the study of genomic disorders with obesity associated as part of the phenotype. In our study, CNVs known to be causal for syndromic obesity were detected in $8.2 \%$ of patients, but we provide evidence for a genetic basis of obesity in as many as $14 \%$ of cases. Overall, our results underscore the genetic heterogeneity in syndromic forms of obesity, which imposes a substantial challenge for diagnosis.
\end{abstract}

Keywords: Chromosomal microarray analysis (CMA), Copy number variations (CNVs), Body mass index (BMI), Intellectual and developmental disabilities (IDDs), Prader-Willi syndrome (PWS), Syndromic obesity

\footnotetext{
* Correspondence: cdangelo@ib.usp.br

${ }^{1}$ Human Genome and Stem Cell Research Center (HUG-CELL), Department of

Genetics and Evolutionary Biology, Institute of Biosciences, University of Sao

Paulo, Rua do Matao no 277, Cidade Universitaria-Butanta, Sao Paulo, SP

05508-090, Brazil

Full list of author information is available at the end of the article
} 


\section{Background}

Obesity is a highly heritable multifactorial disorder defined by a body mass index (BMI) of $\geq 30 \mathrm{~kg} / \mathrm{m}^{2}$, which predisposes to many diseases. Rare and common genetic variants associated with obesity identified to date have increased our understanding of the mechanisms by which obesity develops. Copy number variants (CNVs) in a number of chromosomal regions are known to be involved in highly penetrant and individually rare, both isolated and syndromic forms of obesity [1-3]. The latter describes cases where obesity co-occurs with additional phenotypes (e.g., intellectual and developmental disabilities (IDDs), dysmorphism, congenital anomalies) often arising as part of a distinct syndrome, from which Prader-Willi syndrome (PWS; OMIM \#176270) is a classical example. Until recently, only a few genomic disorders other than PWS were known to contribute to increased risk of obesity, including the known microdeletion syndromes 1p36 (OMIM \#607872), 2q37 (OMIM \#600430), 6q16 (SIM1 gene), 9q34 (OMIM \#610253; EHMT1 gene), 11p14.1 (OMIM \#612469), and 17p11.2 (OMIM \#182290; RAI1 gene).

In recent years, however, numerous unique and rare recurring/overlapping $\mathrm{CNVs}$ have been associated with a syndromic obesity phenotype in patients through the widespread use of chromosomal microarray analysis (CMA) [4-16]. Examples include deletions of chromosome band 2p25.3 that include the MYT1L gene (OMIM \#616521), the recurrent 220-kb deletion of distal 16 p11.2 including the SH2B1 gene (OMIM \#613444), and the recurrent $600-\mathrm{kb} 16 \mathrm{p} 11.2$ proximal deletion (OMIM \#611913, gene unknown). Also, a novel genomic disorder that causes obesity, ID and seizures has been described in children carrying a recurrent unbalance translocation $(8 ; 12)(\mathrm{p} 23.1 ; \mathrm{p} 13.31)$ that duplicates the GNB3 gene [17]. Overlapping 1p21.3 deletions comprising the $D P Y D$ and MIR137 genes have been detected in patients with a phenotype consisting primarily of obesity, ID, and autism spectrum disorder (ASD) $[18,19]$. Small 6q16.1 deletions encompassing the POU $3 F 2$ gene were identified in 10 individuals presenting with obesity, hyperphagia and IDDs [20]. Chromosome 13q34 deletions disrupting the CHAMP1 gene were linked ID, obesity and mild dysmorphism in five adult individuals [21].

Syndromic obesity is recognized as an etiologically heterogeneous group of disorders for which an obesity-related genetic change can be identified but only a few genetic causes have been identified to date. Only one study has examined the etiology of syndromic obesity with CMA in a cohort of 100 patients specifically selected for obesity [22]. In that study, CNVs were regarded either as pathogenic or potentially pathogenic in $22 \%$ of cases, and several novel CNVs for which a defined syndrome has not yet been delineated were uncovered. Herein, we report our experience over the past 5 years using CMA to identify CNVs in 279 patients referred with syndromic obesity. This study adds to the current knowledge of CNVs linked to obesity and provides evidence for association with obesity at new and previously identified candidate loci.

\section{Methods \\ Cohort enrollment and description}

Only patients who tested negative for PWS (methylation analysis of SNURF-SNRPN exon 1 by our laboratory) were included in our study whether or not they had a positive clinical score for PWS. This test population had a mean age of 9 years (range 8 days to 40 years old), $55 \%$ of cases represented by male patients (male/female ratio $=1.2$ ). The 2000 Centers for Disease Control and Prevention (CDC) growth charts (available at https:// www.cdc.gov/growthcharts/) were used to plot weightfor-age, height-for-age, weight-for-height, BMI-for-age, and occipito-frontal head circumference (OFC) [23]. We stratified our cohort into 4 age groups: $(1)$ infants $(n=19)$ $<2$ years old (mean age 13 months; 9 males and 9 females); (2) children ( $n=153)$ aged $2-9$ years (mean age 6 years; 80 males and 73 females); (3) adolescents $(n=98)$ aged 10-19 years (mean age 9 years; 61 males and 37 females); (4) adults $(n=10)>20$ years old (mean age 27 years; 2 males and 8 females In a majority of patients, recognition of excessive weight gain was based on the following: (1) infants, the standard deviation (SD) of weight-for-height Z-scores > 1 (mean + 3.23 SD); (2) children and adolescents, BMI-for-age percentiles $\geq 85$ th (mean 97.4th and 97.9th percentiles, respectively); (3) adults, BMI values $\geq 30 \mathrm{~kg} / \mathrm{m} 2$ (mean $46.7 \mathrm{~kg} / \mathrm{m} 2$ ); In 37 patients, data on weight and/or height were missing but they had a documented diagnosis of overweight or obesity made by attending physicians, and 5 other patients aged $<$ 5 years had hyperphagia with an increase probability of developing obesity. Among a subset of 208 children and adolescents (BMI $\geq 95$ th percentile), we further classified obesity based on the BMI expressed as a percentage above the 95th BMI percentile according to age and sex, as previously described $[24,25]$ : a BMI $100-119 \%$ of the 95th percentile was used to define moderate obesity and a BMI $\geq 120 \%$ of the 95th BMI percentile used to define severe obesity. Extreme BMIs were calculated by multiplying the BMI at the 95th percentile by a factor of 1.1 through 1.9 to derive the $110 \%$ to $190 \%$, for both genders.

\section{Chromosomal microarray analysis}

Any of the following genome-wide array platforms were used according to their availability: CytoSure ISCA v2 4x180K (Oxford Gene Technology, Oxford, UK), SurePrint G3 Human CGH 8x60K (Agilent Technologies, Santa Clara, CA), Affymetrix Mapping $100 \mathrm{~K}$ and $500 \mathrm{~K}$ arrays (Affymetrix, Santa Clara, CA, USA). Most cases (85\%) were investigated using high-density oligonucleotide microarrays 
(4x180K OGT platform). DNA was extracted from peripheral blood using Autopure LS (Gentra Systems, Inc., Minneapolis, MN). Genomic DNA concentration was measured by Nanodrop spectrophotometer (ThermoFisher). Chromosomal microarray testing was performed according to the manufacturers' instructions. In oligonucleotide-based microarrays, two experiments were performed for each patient sample with reversal of the dye labels for the control and test samples, raw data were processed and analyzed using Agilent Feature Extraction and Genomic Workbench software with the statistical algorithm ADM-2 and sensitivity threshold of 6.7. Affymetrix SNP array data was analyzed with the Genotyping Console (GTC) 4.0 software using default settings and a similarly processed reference sample data set. Due to the limited probe coverage, CNVs on chromosome $\mathrm{Y}$ were removed from the analysis. We used the American College of Medical Genetics and Genomics (ACMG) 2011 guidelines for variant interpretation to classify variants in 4 categories: pathogenic CNVs (PCNVs), likely PCNVs, variants of uncertain significance (VUS), and likely benign $\mathrm{CNVs}$ [26]. Healthy and disease variant databases used included the Database of Genomic Variants (DGV, http://dgv.tcag.ca), the Online Mendelian Inheritance in Man (OMIM, https://www.omim.org/) and the DatabasE of genomiC varlation and Phenotype in Humans using Ensembl Resources (DECIPHER, http://decipher.sanger.ac.uk) [27-29]. All genomic breakpoints were based on the human genome build GRCh37 (hg19) (http://genome.ucsc.edu/) [30].

\section{Gene prioritization}

Genes affected by the detected CNVs were compared to a list of genes related to obesity downloaded from the Textmined Hypertension, Obesity and Diabetes candidate gene database (T-HOD) [31] and the Human Genome Epidemiology encyclopedia Navigator (HUGE, https:// phgkb.cdc.gov/PHGKB) [32]. We specifically searched the term "obesity" and retrieved 835 genes annotated in T-HOD and 1920 genes annotated in the HUGE Phenopedia. We also checked the genes affected by CNVs against a list of 370 genes with evidence for playing a role in obesity curated from literature [33] and a list of 940 genes in the CNV morbidity map for IDDs generated from 29,085 cases and 19,584 controls [34].

\section{Results}

Cohort and correlation of PCNVs with specific phenotypes General clinical findings noted in patients are listed in Table 1 (individual descriptions are provided in Table S1 in Additional file 1). Although patients' records were not always complete and clinical comorbidities could not be fully assessed, the most commonly reported features associated with obesity were IDDs, dysmorphism, behavioral phenotypes, hyperphagia, neonatal hypotonia, and language
Table 1 Additional phenotypes of patients with syndromic obesity

\begin{tabular}{lll}
\hline Clinical features & Total cohort (n) & $\begin{array}{l}\text { Patients } \\
\text { with PCNVs (n) }\end{array}$ \\
\hline Intellectual/developmental disabilities & 219 & 55 \\
Craniofacial dysmorphism & 149 & 49 \\
Behavioral problems & 132 & 29 \\
Hyperphagia & 112 & 27 \\
Infantile hypotonia & 88 & 32 \\
Language impairments & 80 & 28 \\
Hands/ft abnormalities & 65 & 21 \\
Abnormal external genitalia & 56 & 20 \\
Eye/vision problems & 51 & 16 \\
Seizures & 31 & 13 \\
Poor motor skills & 29 & 9 \\
Skeletal anomalies & 23 & 9 \\
Brain abnormalities & 19 & 7 \\
Hearing loss & 13 & 6 \\
Cardiac abnormalities & 10 & 4 \\
\hline
\end{tabular}

Prevalence could not be assess as complete phenotypic data was not available

impairments. Hands and feet abnormalities, abnormalities of the external genitalia and eye/vision problems were often reported. Macrocephaly was observed in 92 of 206 (45\%) patients, compared to 11 of 206 (5\%) patients with microcephaly, and tall stature in 40 of 238 (17\%) patients, compared to 22 of 238 (9\%) patients with short stature (Zscores $>$ or $< \pm 2 \mathrm{SD}$ ). No association was found between these growth parameters in cases with and without PCNVs using the Fisher's Exact test (Fig. 1).

In an attempt to determine whether there were phenotypic differences associated with the presence of PCNVs, we compared the frequencies of phenotype pairs segregating together in patients with syndromic obesity caused by PCNVs against those without PCNVs. We constructed a matrix representation (heatmap) of the Chi-square $p$-values between any given pair of phenotypes that co-occurred in patients with and without PCNVs. Out of 133 phenotype pairs that were evaluated (listed in Table S2 in Additional file 2), 12 had significant associations for PCNVs with $p$-values $<0.05$ (Fig. 2a). Next, we constructed using Cytoscape [35] a graphic representation of these phenotype-phenotype associations, where a given pair of phenotype was connected only if they were significant at $p<0.05$, to discover the core phenotype variables in the network (those that overlapped most between pairs). The most highly correlated phenotypes are hypotonia, language impairments, abnormalities of the external genitalia, and eye/vision problems. They also correlated with many additional phenotype variables including seizures, sleep problems, tall stature and hands/feet abnormalities (Fig. 2b). 


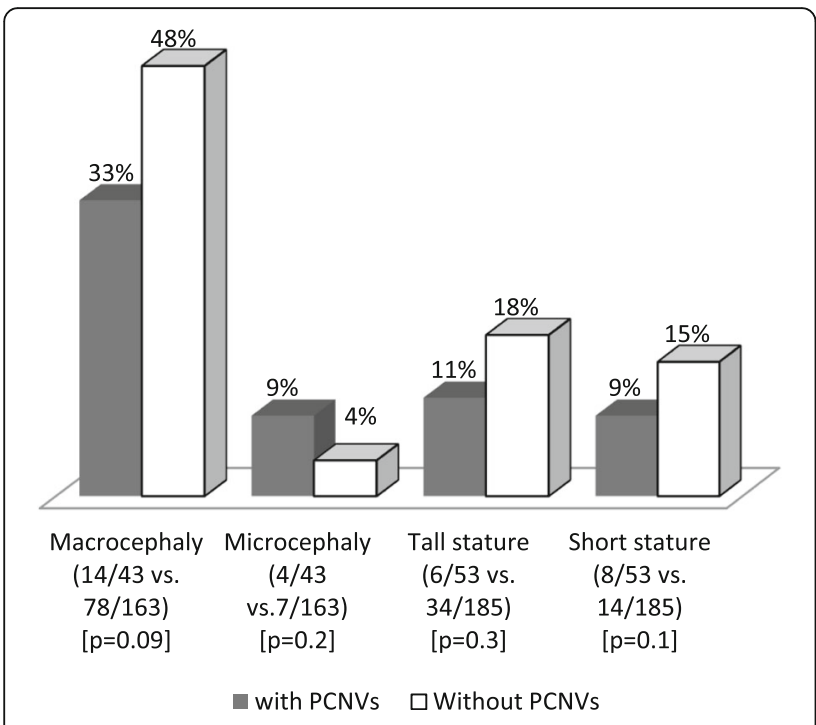

Fig. 1 Comparison of frequencies of each variable among patients with and without PCNVs. The fractions in parentheses indicate the number of cases that manifested the phenotype over the total number of cases with data. $P$-values for the Fisher's exact test are shown. Macro/microcephaly and tall/short stature were defined by the Z-scores $>$ or $< \pm 2$ SD

\section{Characterization of CNVs in patients with syndromic obesity}

Overall, CMA identified clinically relevant genomic imbalances in $22 \%$ of patients, potentially clinically relevant CNVs in $2 \%$, VUS in $5 \%$ and likely benign CNVs in $11 \%$ (Table 2). All clinically relevant results per individual are listed in Table 3. The genomic regions associated with likely PCNVs, VUS and likely benign CNVs are listed in Additional file 3: Table S3.

\section{Pathogenic CNVs}

A total of 68 pathogenic imbalances were detected in 61 patients, the majority of which pathogenic deletions (72\%) and rearrangements smaller than 5-Mb (70\%). 31 of the patients $(6-12,14,15,18,23,28,30-33,35,40-$ $43,46-48,51,52,55,56,58-60)$ had previously been published as separate studies [36-38]. De novo PCNVs were found in 30 patients, whereas only 6 patients inherited a pathogenic deletion or duplication from an apparently unaffected parent, all of which occurring at genomic loci which are known to have reduced penetrance (16p13.11, 16p11.2, and 22q11.2). The inheritance status could not be determined in 25 cases.

In 47 patients (24 novel cases), the PCNVs overlapped with chromosomal regions associated with known genomic disorders, and, among them, 35 patients were detected with PCNVs at 10 loci that were recurrent (same breakpoints) or overlapping in 2 or more unrelated samples (Fig. 3). We found 23 cases with deletions known to cause a syndromic obesity phenotype: 1p36 $(n=5), 1 \mathrm{p} 21.3(n=2), 2 \mathrm{p} 25.3(n=1), 2 \mathrm{q} 37(n=5), 6 \mathrm{q} 16$ $(n=1), 9 q 34.3(n=1), 16 \mathrm{p} 11.2$ breakpoint (BP) $2-3$ $(n=1), 16 \mathrm{p} 11.2 \mathrm{BP} 4-5(n=1)$, and 17p11.2 $(n=5)$. The recurrent translocation $\mathrm{t}(8 ; 12)(\mathrm{p} 23.1 ; \mathrm{p} 13.31)$ found in patient 18 is also known to be involved in the pathogenesis of syndromic obesity. In addition, 2 rare deletions at chromosomes 13q12.3 (patient 24) and 19p13.12 (patient 37) overlapped with deletions of different sizes in patients from the literature and the DECIPHER database who were obese (Fig. 4). Mapping of the shortest region of overlap (SRO) in these cases exposed a 660-kb interval at 13q12.3 (chr13:30,880,255-31,540,272 bp, hg19; Fig. 4a) comprising 5 genes (KATNAL1, LINC00426, HMGB1, USPL1, ALOX5AP, and MEDAG), and a 440$\mathrm{kb}$ interval at 19p13.12 (chr19:15,052,889$15,492,848$ bp, hg19; Fig. 4b) comprising 9 genes (SLC1A6, CCDC105, CASP14, SYDE1, ILVBL NOTCH3, $E P H X 3, B R D 4$, and $A K A P 8)$.

In other 14 patients, the PCNVs did not overlap with a known genomic imbalance disorder but were de novo in 10 cases or of unknown origin (the remainder), and involved large and complex chromosomal imbalances. Among these, we found 4 PCNVs overlapping with previously identified obesity candidate loci (Fig. 5): a novel 22.3-Mb duplication of 3q11.2q13.31 (patient 49), a novel 11.6-Mb duplication of 13q11q12.3 (patient 53), a novel $8.5-\mathrm{Mb}$ deletion of $13 \mathrm{q} 33.2 \mathrm{q} 34$ (patient 57 ), and a $1-\mathrm{Mb}$ duplication of band 14q11.2 (patient 58). Notably, the duplication region at $13 q 11 q 12.3$ also overlaps with a smaller duplication present in our patient 19, who possessed a second large $\mathrm{CNV}$ at the 8p23.1 locus. The extent of overlap among our cases with those previously described CNVs is of about 2-Mb in band $3 q 13.31$ (chr3:113,924,534-115,890,384 bp, hg19; Fig. 5a), 1.2-Mb in band 13q12.12 (chr13:23,706,634-24,910,765 bp, hg19; Fig. 5b), 2.4-Mb in band 13q34 (chr13:112,725,394$115,092,648$ bp, hg19; Fig. 5c), and 827-kb in band 14q11.2 (chr14:21,424,185-22,250,879 bp, hg19; Fig. 5d). Candidate genes at these intervals are proposed in the discussion.

\section{Likely pathogenic CNVs}

CNVs detected in 6 additional patients were classified as potentially clinically significant, including a de novo 340$\mathrm{kb}$ duplication at 16p13.2 comprising the USP7 gene implicated in a known deletion syndrome (OMIM \#616863), a $482-\mathrm{kb}$ paternally inherited duplication of $17 \mathrm{q} 11.2$ partially overlapping the gene for neurofibromatosis type I (NF1), and an 1.1-Mb paternally inherited 20q11.2 duplication upstream of the ASXL1 gene with a likely role in 20q11.2 duplication syndrome [39]. The inheritance of 3 other CNVs could not be determined. The $3 \mathrm{CNVs}$ were a 489-kb duplication in 21q22.13 including the ID gene DYRK1A (OMIM \#614104), a 703-kb deletion in 7q31.1 affecting the $I M M P 2 L-D O C K 4$ gene region implicated in 

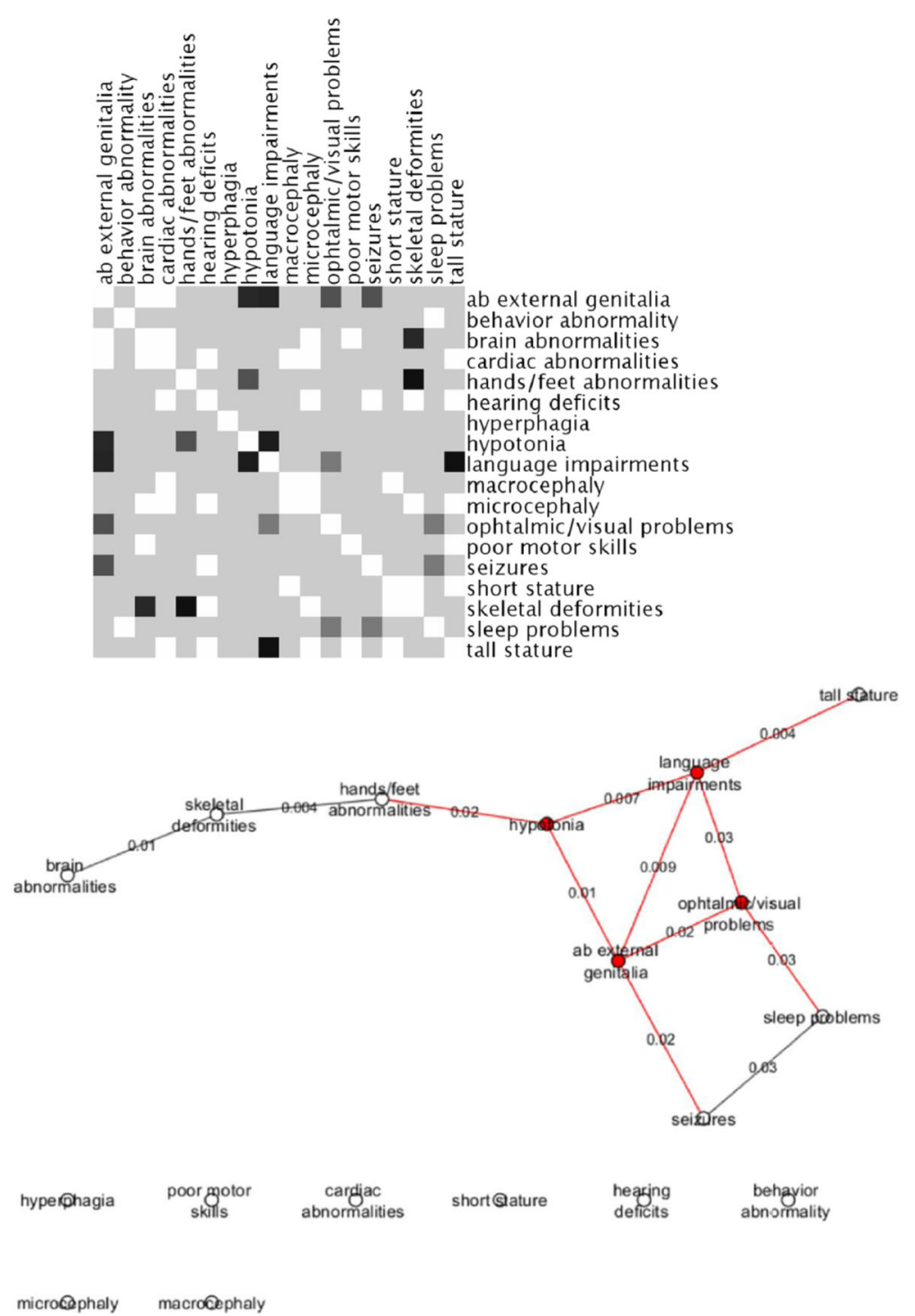

Fig. 2 The heat map constructed from the $P$-values for the Chi-square statistic test between pairs of phenotypes observed in patients with PCNVs against those without PCNVs is shown at the top, where $P$-values $<0.05$ are represented by small darker gray or black squares and larger values by light gray squares (white squares indicate null values or the absence of association between a given pair of phenotype). Graphical representation of the phenotype network generated using Cytoscape is shown at the bottom, where phenotypes (nodes) are interconnected (edges) if they had significant associations for PCNVs at $P<0.05$. The resulting network has 10 interconnected phenotypes and 8 not connected phenotype variables. Red nodes and edges highlight the most highly connected phenotypes and their interactions in the network

IDDs [40], and an intragenic deletion of the ASTN2 gene at 9q33.1 which has also been implicated in susceptibility to IDDs [41].

\section{Variants of uncertain significance}

In 15 patients, we detected CNVs that were classified as VUS. Of these, 2 were de novo events: a 222-kb 12q21.32 duplication including the CEP290 gene whose mutations cause Bardet-Biedl syndrome (BBS14; OMIM \#615991), but which was not associated with previously reported pathogenicity, and a 385-kb 6q27 duplication affecting only three non-coding RNAs found in a patient who inherited a second large CNV. There were 3 VUS inherited from asymptomatic parents intersecting with genes within $\mathrm{CNVs}$ that have previously been implicated with disorders, such as CACNA2D1 with epilepsy and ID [42], and MACROD2 and LINGO2 with autism [43, 44]. Furthermore, 6 VUS (3 inherited and 3 unknown) contained morbid OMIM genes, including NFIA, MPZ, PARK2, DPP6, and KANK1. Additionally, 3 other cases (2 females and 1 male) inherited large chromosome $\mathrm{X}$ duplications from carrier mothers spanning several morbid OMIM genes but with no 
Table 2 Overall findings of microarray testing

\begin{tabular}{lll}
\hline & Number & $\%$ \\
\hline Total number of cases & 279 & - \\
With imbalances & 112 & 40 \\
With pathogenic CNVs & 61 & 22 \\
With Known syndromes & 47 & 17 \\
Pathogenic imbalances ${ }^{\text {a }}$ & 68 & - \\
Del & 49 & 72 \\
Dup & 19 & 28 \\
$>5$ Mb & 21 & 31 \\
De novo & 30 & 49 \\
Inherited & 6 & 10 \\
Unknown & 19 & 31 \\
Not maternal & 6 & 10 \\
With likely pathogenic CNVs & 6 & 2 \\
With CNVs of uncertain significance & 15 & 5 \\
With likely benign CNVs & 30 & 11 \\
\hline
\end{tabular}

aPathogenic imbalances included 45 simple deletions or duplications, 4 unbalanced translocations, an insertional translocation, and 6 other complex rearrangements believed to have been formed from the same rearrangement. In the remaining 5 patients, the rearrangements were associated with a second-site CNV arisen apparently independent which were classified as benign or of uncertain significance

evidence for triplosensitivity phenotypes as determined by the ClinGen Dosage Sensitive Map (http://www.ncbi.nlm.nih.gov/projects/dbvar/clingen/) [45].

\section{Likely benign CNVs}

We also observed 30 patients with CNVs that might represent benign variants. The observed CNVs were most often duplications and $<300-\mathrm{kb}$ in size. In all cases where it was possible to ascertain the parental status, variants were inherited from an asymptomatic parent. We found relevant genes lying within some of these CNVs. Examples of such genes include: PTEN in which mutations cause many different disorders including macrocephaly/autism syndrome (OMIM \#605309); VPS13B whose mutations cause Cohen syndrome characterized by truncal obesity, joint hypermobility and a pigmentary retinopathy $(\mathrm{COH} 1$; OMIM \#216550); CIDEA (OMIM 604440) with a role in regulating energy balance and adiposity; ULK4 crucial to brain development with CNVs being identified as risk factor in schizophrenia [46]; KATNAL2 implicated as susceptibility gene of autism [47].

\section{Identification of candidate genes involved in obesity susceptibility}

A total of 2684 genes were affected by the detected CNVs. Among these, 234 genes had some previously reported connection to obesity as determined by the overlap with genes from the T-HOD and HUGE database, as well as a gene list curated from literature [33], and 172 overlapped with genes listed in the CNV morbidity map for IDDs [34] (367 genes in total). Of particular interest are 87 genes that were intersected by different gene sets (Fig. 6). Notably, several known and candidate genes that have previously been implicated in syndromic obesity were retrieved by this candidate gene approach, including SIM1 [48], SH2B1 [49], PTBP2 [38], PRLH and CAPN10 [50], ACP1 and TMEM18 [12], EHMT1 [51], and GNB3 [17]. Thus, these genes were considered as more likely to have a role in obesity susceptibility. Pathways analysis in Cytoscape using the plugin Reactome FI showed that the majority of these genes were related to metabolic pathways and small molecule metabolic process.

\section{PCNV rates by gender, age and level of obesity in children and adolescents}

For the purpose of this study, 208 obese children and adolescents with a BMI $\geq 95$ th percentile were considered based on a percentage above the 95th BMI percentile as moderately $(<120 \%$ of the 95 th percentile) and severely ( $\geq 120 \%$ of the 95 th percentile) obese (Fig. $7 \mathrm{a}$ ). This sample consisted predominantly of males $(n=123$ or $59 \%$ ) and children aged $2-9$ years $(n=125$ or $60 \%)$. The majority of patients were classified as severely obese ( $n=160$ or $77 \%)$. The prevalence of severe obesity was higher than moderate obesity for both gender and age groups (Fig. 7b) but there were statistically significantly more males than females with severe obesity (105 males, 55 females; Fisher's Exact test, $p<0.01)$; this sex-related difference in obesity was observed in children but not in adolescents aged 10-19 years (Additional file 4: Table S4). Although a higher frequency of PCNVs was observed in females $(n=23$ or $27 \%)$ compared with males $(n=24$ or $20 \%$ ), no statistically significantly differences were observed in the frequencies when compared by gender, age at the time of testing, and obesity severity (Fig. 7c and d; Additional file 5: Table S5). In boys, the prevalence of PCNVs was greater in the severe obesity group (20\% vs. $17 \%)$, particularly among children aged $2-9$ years ( $18 \%$ vs. $11 \%)$, but was similar at adolescence (22\%). In girls, the prevalence of PCNVs was greater among the moderate obesity group (35\% vs. $30 \%)$, particularly among children aged $2-9$ years (35\% vs. $25 \%$ ), and correlated inversely in adolescents ( $26 \%$ severe vs. $20 \%$ moderate obesity).

\section{Discussion}

This is the second study describing the use of CMA in patients ascertained for syndromic obesity, the largest published to date and also the first in a Brazilian cohort. We identified PCNVs in $22 \%$ of patients (68 pathogenic events in $61 / 279$ subjects; Table 3), which is similar to the yield reported by Vuillaume et al. in microarray studies with 100 patients with syndromic obesity [22]. The prevalence of 
Table 3 Pathogenic copy number variations (PCNVs) detected in 279 patients with syndromic obesity

\begin{tabular}{|c|c|c|c|c|c|c|c|c|c|c|}
\hline $\begin{array}{l}\text { Case } \\
\text { number }\end{array}$ & Age & Gender & $\begin{array}{l}\text { Weight } \\
\text { Status }\end{array}$ & $\begin{array}{l}\text { CNV } \\
\text { Type }\end{array}$ & Cytoband & $\begin{array}{l}\text { Genome } \\
\text { Coordinate }\end{array}$ & Size & Origin & $\begin{array}{l}\text { Clinical } \\
\text { significance }\end{array}$ & RefSeq genes \\
\hline \multicolumn{11}{|c|}{ Genomic imbalance disorders } \\
\hline P1 & $5 y$ & $\mathrm{~F}$ & $\begin{array}{l}\text { Referred as } \\
\text { obese }\end{array}$ & Del & $1 \mathrm{p} 36.33$ & $\begin{array}{l}734595- \\
1970865\end{array}$ & 1236270 & Unk & $\begin{array}{l}1 \mathrm{p} 36 \text { terminal } \\
\text { deletion }\end{array}$ & $\begin{array}{l}\text { KLHL17, AGRN, TAS1R3, }{ }^{*} D V L 1, V W A 1 \\
\text { MMP23B, GABRD }\end{array}$ \\
\hline \multirow[t]{2}{*}{ P2 } & $34 y$ & $\mathrm{~F}$ & BMI 59.1 & Del & 1 p36.33 & $\begin{array}{l}734595- \\
2223317\end{array}$ & 1488722 & Unk & $\begin{array}{l}1 \mathrm{p} 36 \text { terminal } \\
\text { deletion, } \\
\text { complex }\end{array}$ & 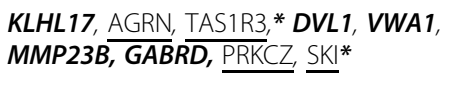 \\
\hline & & & & Dup & $\begin{array}{l}\text { 1p36.33 } \\
\text { p36.32 }\end{array}$ & $\begin{array}{l}2225679- \\
2694799\end{array}$ & 469120 & & & - \\
\hline P3 & $14 y$ & $\mathrm{~F}$ & $\begin{array}{l}\text { BMI } 26.8 \\
\text { (93.3th) }\end{array}$ & Del & $\begin{array}{l}\text { 1p36.33 } \\
\text { p36.32 }\end{array}$ & $\begin{array}{l}794592- \\
2377269\end{array}$ & 1582677 & $\begin{array}{l}\text { De } \\
\text { novo }\end{array}$ & $\begin{array}{l}1 \mathrm{p} 36 \text { terminal } \\
\text { deletion }\end{array}$ & 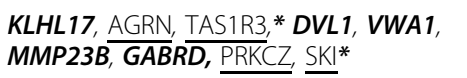 \\
\hline P4 & $6 y$ & $\mathrm{~F}$ & $\begin{array}{l}\text { BMI } 19.7 \\
\text { (96.8th) }\end{array}$ & Del & $\begin{array}{l}\text { 1p36.33 } \\
\text { p36.32 }\end{array}$ & $\begin{array}{l}734595- \\
3531040\end{array}$ & 2796445 & Unk & $\begin{array}{l}1 \mathrm{p} 36 \text { terminal } \\
\text { deletion }\end{array}$ & 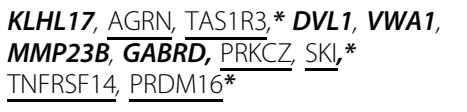 \\
\hline P5 & $11 y$ & M & $\begin{array}{l}\text { BMI } 30.8 \\
\text { (99th) }\end{array}$ & Del & $\begin{array}{l}1 \mathrm{p} 36.31 \\
\text { p36.22 }\end{array}$ & $\begin{array}{l}6204969- \\
9433118\end{array}$ & 3228149 & Unk & $\begin{array}{l}1 \text { p36 interstitial } \\
\text { deletion }\end{array}$ & CAMTA1， $\underline{\text { PER3 }}, \underline{\text { UTS2 }}$, RERE, $\underline{\text { H6PD }}{ }^{*}$ \\
\hline P6 & $15 y$ & $\mathrm{~F}$ & $\begin{array}{l}\text { BMI } 37.1 \\
\text { (99.1th) }\end{array}$ & Del & $\begin{array}{l}1 \mathrm{p} 22.1 \\
\mathrm{p} 21.2\end{array}$ & $\begin{array}{l}93919217- \\
99846176\end{array}$ & 5926959 & $\begin{array}{l}\text { De } \\
\text { novo }\end{array}$ & 1 p21.3 deletion & $\underline{\mathrm{F} 3}, \underline{\mathrm{PTBP} 2}, *{ }^{*} D P Y D, M I R 137$ \\
\hline P7 & $8 y$ & $\mathrm{~F}$ & $\begin{array}{l}\text { BMI } 33.2 \\
\text { (99.7th) }\end{array}$ & Del & $\begin{array}{l}1 \mathrm{p} 21.3 \\
\mathrm{p} 13.3\end{array}$ & $\begin{array}{l}95696444- \\
107755879\end{array}$ & 12059435 & Unk & 1 p21.3 deletion & $\begin{array}{l}\text { PTBP2, }{ }^{*} \text { DPYD, MIR137, VCAM1, } \\
\text { COL11A1, AMY2B, AMY2A, AMY1A* }\end{array}$ \\
\hline P8 & $6 y$ & M & $\begin{array}{l}\text { BMI } 28.8 \\
\text { (99.9th) }\end{array}$ & Dup & $1 q 21.1$ & $\begin{array}{l}146074084- \\
147828029\end{array}$ & 1753945 & Unk & $\begin{array}{l}\text { 1q21.1 distal } \\
\text { duplication }\end{array}$ & PRKAB2, CHD1L, GJA5, GJA8 \\
\hline P9 & $7 y$ & $\mathrm{~F}$ & $\begin{array}{l}\text { BMI 22.6 } \\
\text { (98.2th) }\end{array}$ & Del & $2 p 25.3$ & $\begin{array}{l}63452- \\
3215593\end{array}$ & 3152141 & $\begin{array}{l}\text { De } \\
\text { novo }\end{array}$ & $\begin{array}{l}\text { 2p25.3 terminal } \\
\text { deletion }\end{array}$ & $\underline{\mathrm{ACP} 1}, *{ }^{*}{ }^{\mathrm{TMEM} 18}, *$ SNTG2, TPO, ${ }^{*}$ MYT1L \\
\hline P10 & $8 y$ & $\mathrm{~F}$ & $\begin{array}{l}\text { BMI } 29.1 \\
\text { (99.5th) }\end{array}$ & Del & $\begin{array}{l}2 q 37.2 \\
q 37.3\end{array}$ & $\begin{array}{l}237220842- \\
242995835\end{array}$ & 5774993 & $\begin{array}{l}\text { De } \\
\text { novo }\end{array}$ & $\begin{array}{l}\text { 2q37 terminal } \\
\text { deletion }\end{array}$ & $\begin{array}{l}\text { PRLH, } \\
\text { LARRFIP1， PER2, HDAC4, GPC1, }{ }^{*} \\
\text { CAPN } 10,{ }^{*} \text { GPR } 35,{ }^{*} \text { KIF1A, PASK, STK } 25^{*}\end{array}$ \\
\hline P11 & $21 y$ & M & $\begin{array}{l}\text { Referred as } \\
\text { obese }\end{array}$ & Del & $\begin{array}{l}2 q 37.2 \\
q 37.3\end{array}$ & $\begin{array}{l}236854160- \\
242995835\end{array}$ & 6141675 & Unk & $\begin{array}{l}\text { 2q37 terminal } \\
\text { deletion }\end{array}$ & $\begin{array}{l}\text { AGAP1, PRLH, LRRFIP1, PER2, HDAC4, } \\
\text { GPC1, }{ }^{*} \text { CAPN10, }{ }^{*} \text { GPR } 35,{ }^{*}{ }^{*} \text { KIF1A, PASK, }\end{array}$ \\
\hline \multirow[t]{2}{*}{ P12 } & $10 y$ & M & $\begin{array}{l}\text { BMI } 29.8 \\
\text { (99th) }\end{array}$ & Del & $\begin{array}{l}2 q 37.2 \\
q 37.3\end{array}$ & $\begin{array}{l}236944801- \\
243014630\end{array}$ & 6069829 & $\begin{array}{l}\text { De } \\
\text { novo }\end{array}$ & $\begin{array}{l}2 \text { q37 terminal } \\
\text { deletion, } \\
\text { complex }\end{array}$ & $\begin{array}{l}\text { AGAP1, PRLH, LRRFIP1, PER2, HDAC4, } \\
\text { GPC1, }{ }^{*} \text { CAPN10, }{ }^{*} \text { GPR3 } 35,{ }^{*} \text { KIF1A, PASK, }\end{array}$ \\
\hline & & & & Dup & $\begin{array}{l}2 q 37.1 \\
q 37.2\end{array}$ & $\begin{array}{l}235090417- \\
236802930\end{array}$ & 1712513 & & & - \\
\hline \multirow[t]{2}{*}{ P13 } & $9 y$ & $\mathrm{~F}$ & $\begin{array}{l}\text { BMI } 24.7 \\
\text { (98.2th) }\end{array}$ & Del & $\begin{array}{l}2 q 37.1 \\
q 37.3\end{array}$ & $\begin{array}{l}234850276- \\
243028335\end{array}$ & 8178059 & Unk & $\begin{array}{l}\text { 2q37 terminal } \\
\text { deletion, } \\
\text { complex }\end{array}$ & $\begin{array}{l}\text { TRPM8, AGAP1, PRLH, LRRFIP1, PER2, } \\
\text { HDAC4, GPC1, }{ }^{*} \frac{\text { CAPN } 10,{ }^{*} \text { GPR } 355^{*}}{{ }^{*}} \\
\text { KIF1A, }\end{array}$ \\
\hline & & & & Dup & $2 q 37.1$ & $\begin{array}{l}233867403- \\
234794816\end{array}$ & 927413 & & & $\underline{\text { UTG1A1 }}^{*}$ \\
\hline \multirow[t]{2}{*}{ P14 } & $5 y$ & $\mathrm{~F}$ & $\begin{array}{l}\text { BMI } 24.0 \\
\text { (99.7th) }\end{array}$ & Del & $2 q 37.3$ & $\begin{array}{l}240880562- \\
242948060\end{array}$ & 2067498 & Unk & $\begin{array}{l}\text { Unbalanced } \\
\text { translocation }\end{array}$ & 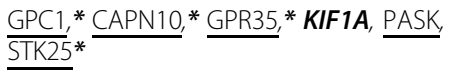 \\
\hline & & & & Dup & $17 q 25.3$ & $\begin{array}{l}78709250- \\
81036261\end{array}$ & 2327011 & & & $\begin{array}{l}\text { RPTOR, ACTG1, GCGR, * PCYT2, FASN, }{ }^{*} \\
\text { CSNK1D, UTS2R }\end{array}$ \\
\hline P15 & $10 y$ & M & $\begin{array}{l}\text { BMI } 31.0 \\
\text { (99.3th) }\end{array}$ & Del & $\begin{array}{l}6 \mathrm{q} 16.1 \\
\mathrm{q} 21\end{array}$ & $\begin{array}{l}95836632- \\
108010940\end{array}$ & 12174308 & $\begin{array}{l}\text { De } \\
\text { novo }\end{array}$ & $6 q 16$ deletion & $\begin{array}{l}\text { KLHL32, POU3F2, MCHR2, }{ }^{*} \text { SIM1, * GRIK2, } \\
\text { LIN28B, }\end{array}$ \\
\hline P16 & $15 y$ & $\mathrm{~F}$ & $\begin{array}{l}\text { BMl } 40.5 \\
\text { (99.4th) }\end{array}$ & Del & $7 q 11.23$ & $\begin{array}{l}72420782- \\
74985644\end{array}$ & 2564862 & Unk & 7q11.23 deletion & 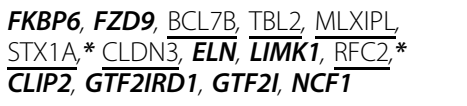 \\
\hline P17 & $6 y$ & M & $\begin{array}{l}\text { BMI } 30.6 \\
\text { (99.9th) }\end{array}$ & Del & $7 q 11.23$ & $\begin{array}{l}72437606- \\
75053787\end{array}$ & 2616181 & Unk & 7q11.23 deletion & 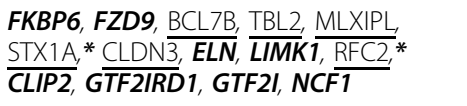 \\
\hline P18 & $6 y$ & M & $\begin{array}{l}\text { BMI } 33.4 \\
\text { (99.9th) }\end{array}$ & Del & $\begin{array}{l}8 p 23.3 \\
\text { p23.1 }\end{array}$ & $\begin{array}{l}176464- \\
7786759\end{array}$ & 7610295 & $\begin{array}{l}\text { De } \\
\text { novo }\end{array}$ & $\begin{array}{l}\text { Unbalanced } \\
\text { translocation }\end{array}$ & $\begin{array}{l}\text { CLN8, CSMD1, DEFA1, } * \text { DEFB103A, } \\
\text { DEFB103B, DEFB104A, DEFB106A, } \\
\text { DEFB105A, DEFB107A, DEFB4A }\end{array}$ \\
\hline
\end{tabular}


Table 3 Pathogenic copy number variations (PCNVs) detected in 279 patients with syndromic obesity (Continued)

\begin{tabular}{|c|c|c|c|c|c|c|c|c|c|c|}
\hline $\begin{array}{l}\text { Case } \\
\text { number }\end{array}$ & Age & Gender & $\begin{array}{l}\text { Weight } \\
\text { Status }\end{array}$ & $\begin{array}{l}\text { CNV } \\
\text { Type }\end{array}$ & Cytoband & $\begin{array}{l}\text { Genome } \\
\text { Coordinate }\end{array}$ & Size & Origin & $\begin{array}{l}\text { Clinical } \\
\text { significance }\end{array}$ & RefSeq genes \\
\hline & & & & Dup & $\begin{array}{l}\text { 12p13.33 } \\
\text { p13.31 }\end{array}$ & $\begin{array}{l}204618- \\
8309473\end{array}$ & 8104855 & & & 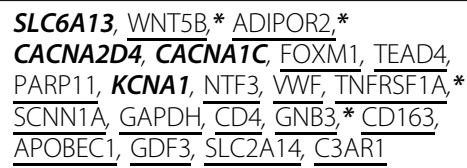 \\
\hline \multirow[t]{2}{*}{ P19 } & $6 y$ & M & $\begin{array}{l}\text { BMI 25.8 } \\
\text { (99.8th) }\end{array}$ & Dup & $8 p 23.1$ & $\begin{array}{l}8054556- \\
11985356\end{array}$ & 3930800 & $\begin{array}{l}\text { Not } \\
\text { mat }\end{array}$ & $\begin{array}{l}\text { 8p23.1 } \\
\text { duplication }\end{array}$ & $\begin{array}{l}\text { CLDN23, MFHAS1, PPP1R3B, }, \text { LOC157273, } \\
\text { TNKS, }{ }^{*} \text { MSRA, }{ }^{*} \text { SOX7, MTMR } 9,{ }^{*} \text { BLK } \\
\text { GATA4, }{ }^{*} \text { NEIL2, FDFT1, }{ }^{*} \text { CTSB }\end{array}$ \\
\hline & & & & Dup & $13 q 12.12$ & $\begin{array}{l}23706634- \\
24910765\end{array}$ & 1204131 & $\begin{array}{l}\text { Not } \\
\text { mat }\end{array}$ & Uncertain & SGCG," SACS, MIPEP \\
\hline P20 & $11 y$ & $\mathrm{~F}$ & $\begin{array}{l}\text { BMI 26.7 } \\
\text { (97.6th) }\end{array}$ & Del & $\begin{array}{l}9 p 24.3 \\
\text { p22.3 }\end{array}$ & $\begin{array}{l}40910- \\
14304973\end{array}$ & 14264063 & $\begin{array}{l}\text { De } \\
\text { novo }\end{array}$ & $\begin{array}{l}9 p \text { terminal } \\
\text { deletion }\end{array}$ & $\begin{array}{l}\text { KANK1, DMRT1, SMARCA2, VLDLR, } \\
\text { GLIS3, JAK2, } \\
\text { PTPRD }^{*}\end{array}$ \\
\hline P21 & $12 \mathrm{~m}$ & $\mathrm{~F}$ & $\begin{array}{l}\text { Weight-for } \\
\text { Height }+1 \text { sd }\end{array}$ & Del & $\begin{array}{l}9 p 24.3 \\
\text { p22.3 }\end{array}$ & $\begin{array}{l}204149- \\
15260439\end{array}$ & 15056290 & $\begin{array}{l}\text { De } \\
\text { novo }\end{array}$ & $\begin{array}{l}9 p \text { terminal } \\
\text { deletion }\end{array}$ & 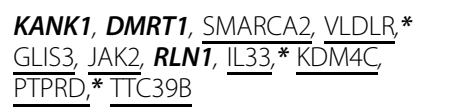 \\
\hline P22 & $17 y$ & M & $\begin{array}{l}\text { BMI } 28.7 \\
\text { (95.7th) }\end{array}$ & Del & $\begin{array}{l}9 p 24.3 \\
\text { p22.3 }\end{array}$ & $\begin{array}{l}201149- \\
8807593\end{array}$ & 8606444 & Unk & $\begin{array}{l}9 p \text { terminal } \\
\text { deletion }\end{array}$ & 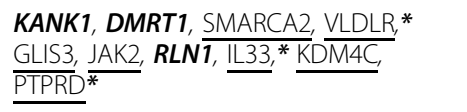 \\
\hline P23 & $9 y$ & $\mathrm{~F}$ & $\begin{array}{l}\text { BMI } 31.1 \\
\text { (99.5th) }\end{array}$ & Del & $9 q 34.3$ & $\begin{array}{l}140665414- \\
141018984\end{array}$ & 353570 & $\begin{array}{l}\text { De } \\
\text { novo }\end{array}$ & 9q34.3 deletion & EHMT1, $*$ CACNA1B \\
\hline P24 & $14 y$ & M & $\begin{array}{l}\text { BMI } 28.7 \\
\text { (97.6th) }\end{array}$ & Del & $\begin{array}{l}13 q 12.3 \\
q 13.1\end{array}$ & $\begin{array}{l}29081250- \\
33529310\end{array}$ & 4448060 & $\begin{array}{l}\text { De } \\
\text { novo }\end{array}$ & $13 q 12.3$ deletion & $\begin{array}{l}\text { POMP, SLC46A3, MTUS2, SLC7A1, UBL3, } \\
\text { KATNAL1, LINCO0426, HMGB1, } \\
\text { ALOX5APP }^{*} \text { RXFP2, BRCA2** }\end{array}$ \\
\hline P25 & $8 y$ & M & $\begin{array}{l}\text { BMI } 25.4 \\
\text { (99.2th) }\end{array}$ & Del & $15 q 11.2$ & $\begin{array}{l}22729423- \\
23086969\end{array}$ & 357546 & Unk & $\begin{array}{l}15 q 11.2 \\
\text { microdeletion }\end{array}$ & NIPA1, NIPA2, CYFIP1 \\
\hline P26 & $10 y$ & $\mathrm{~F}$ & $\begin{array}{l}\text { BMI 22.1 } \\
\text { (93.4th) }\end{array}$ & Del & $\begin{array}{l}\text { 16p13.12 } \\
\text { p13.11 }\end{array}$ & $\begin{array}{l}14780302- \\
16400774\end{array}$ & 1620472 & Pat & 16p13.1 deletion & $\underline{\mathrm{NDE} 1}, \underline{\mathrm{MYH} 11}, *$ ABCC1,$\underline{\mathrm{ABCC} 6}$ \\
\hline P27 & $4 y$ & $\mathrm{~F}$ & Hyperphagia & Dup & $\begin{array}{l}16 p 13.12 \\
\text { p13.11 }\end{array}$ & $\begin{array}{l}14796004- \\
16586941\end{array}$ & 1790937 & Pat & $\begin{array}{l}\text { 16p13.1 } \\
\text { duplication }\end{array}$ & NDE1, $M Y H 11, *$ ABCC1, \\
\hline P28 & $7 y$ & $F$ & $\begin{array}{l}\text { BMI } 20.5 \\
\text { (96.6th) }\end{array}$ & Del & $16 \mathrm{p} 11.2$ & $\begin{array}{l}28843754- \\
29044850\end{array}$ & 201096 & $\begin{array}{l}\text { De } \\
\text { novo }\end{array}$ & $\begin{array}{l}\text { 16p11.2 (BP 2-3) } \\
\text { deletion }\end{array}$ & $\begin{array}{l}\text { ATXN2L } \\
\text { ATP2A1 }\end{array} * \frac{\text { TUFM, }}{\text { SPNS1 }} *$ MIR4721,, SH2B1, \\
\hline P29 & $12 y$ & M & $\begin{array}{l}\text { BMI 30.4 } \\
\text { (98.8th) }\end{array}$ & Del & $16 p 11.2$ & $\begin{array}{l}29592751- \\
30197466\end{array}$ & 604715 & $\begin{array}{l}\text { Not } \\
\text { mat }\end{array}$ & $\begin{array}{l}\text { 16p11.2 (BP 4-5) } \\
\text { deletion }\end{array}$ & $\begin{array}{l}\text { QPRT, PRRT2, SEZ6L2, DOC2A, ALDOA, } \\
\text { TBX6, MAPK3 }\end{array}$ \\
\hline P30 & $8 y$ & M & $\begin{array}{l}\text { Referred as } \\
\text { obese }\end{array}$ & Dup & $16 p 11.2$ & $\begin{array}{l}29592751- \\
30197466\end{array}$ & 604715 & Pat & $\begin{array}{l}\text { 16p11.2 (BP 4-5) } \\
\text { duplication }\end{array}$ & $\begin{array}{l}\text { QPRT, PRRT2, SEZ6L2, DOC2A, ALDOA, } \\
\text { TBX6, MAPK3 }\end{array}$ \\
\hline P31 & $11 y$ & M & $\begin{array}{l}\text { BMI } 42.2 \\
\text { (99.7th) }\end{array}$ & Del & $17 p 11.2$ & $\begin{array}{l}17006987- \\
20171357\end{array}$ & 3164370 & $\begin{array}{l}\text { De } \\
\text { novo }\end{array}$ & 17p11.2 deletion & $\begin{array}{l}\text { COPS3, NT5M, MED9, PEMT,* RAl1, } \\
\text { SREBF1, * ATPAF2, DRG2, SMCR8, } \\
\text { MFAP4, SLC47A1, ALDH3A2, SPECC1 }\end{array}$ \\
\hline P32 & $6 y$ & M & $\begin{array}{l}\text { BMI } 31.2 \\
\text { (99.9th) }\end{array}$ & Del & $17 p 11.2$ & $\begin{array}{l}16757563- \\
20395535\end{array}$ & 3637972 & $\begin{array}{l}\text { Not } \\
\text { mat }\end{array}$ & 17p11.2 deletion & $\begin{array}{l}\text { TNFRSF13B, COPS3, NT5M, MED9, } \\
\text { PEMT,* RAI1, SREBF1, * ATPAF2,, DRG2, } \\
\text { SMCR8, MFAP4, SLC47A1, ALDH3A2, } \\
\text { SPECC1 }\end{array}$ \\
\hline P33 & $7 y$ & $\mathrm{~F}$ & $\begin{array}{l}\text { BMI } 21.9 \\
\text { (97.9th) }\end{array}$ & Del & 17p11.2 & $\begin{array}{l}16603145- \\
20395535\end{array}$ & 3792390 & $\begin{array}{l}\text { De } \\
\text { novo }\end{array}$ & 17p11.2 deletion & $\begin{array}{l}\text { TNFRSF13B, COPS3, NT5M, MED9, } \\
\overline{\text { PEMT,* RAl1, SREBF1, * ATPAF2, DRG2, }} \\
\text { SMCR8, MFAP4, SLC47A1, ALDH3A2, } \\
\text { SPECC1 }\end{array}$ \\
\hline P34 & $8 y$ & F & $\begin{array}{l}\text { BMI } 25.9 \\
\text { (98.9th) }\end{array}$ & Del & 17p11.2 & $\begin{array}{l}16603145- \\
20395535\end{array}$ & 3792390 & Unk & 17p11.2 deletion & $\begin{array}{l}\text { TNFRSF13B, COPS3, NT5M, MED9, } \\
\overline{\text { PEMT,* RAl1, SREBF1, }}{ }^{*} \text { ATPAF2, DRG2, } \\
\text { SMCR8, MFAP4, SLC47A1, ALDH3A2, } \\
\text { SPECC1 }\end{array}$ \\
\hline P35 & $10 y$ & M & $\begin{array}{l}\text { BMI } 28.1 \\
\text { (99th) }\end{array}$ & Del & $17 p 11.2$ & $\begin{array}{l}16603145- \\
20463399\end{array}$ & 3860254 & $\begin{array}{l}\text { De } \\
\text { novo }\end{array}$ & 17p11.2 deletion & $\begin{array}{l}\text { TNFRSF13B, COPS3, NT5M, MED9, } \\
\text { PEMT,* RAl1, SREBF1, * ATPAF2, DRG2, } \\
\text { SMCR8, MFAP4, SLC47A1, ALDH3A2, } \\
\text { SPECC1 }\end{array}$ \\
\hline P36 & $9 y$ & M & $\begin{array}{l}\text { BMI 22.2 } \\
\text { (96.8th) }\end{array}$ & Dup & $\begin{array}{l}17 q 21.31 \\
q 21.32\end{array}$ & $\begin{array}{l}40993738- \\
45166786\end{array}$ & 4173048 & $\begin{array}{l}\text { De } \\
\text { novo }\end{array}$ & $\begin{array}{l}17 q 21.3 \\
\text { duplication }\end{array}$ & 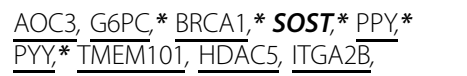 \\
\hline
\end{tabular}


Table 3 Pathogenic copy number variations (PCNVs) detected in 279 patients with syndromic obesity (Continued)

\begin{tabular}{|c|c|c|c|c|c|c|c|c|c|c|}
\hline $\begin{array}{l}\text { Case } \\
\text { number }\end{array}$ & Age & Gender & $\begin{array}{l}\text { Weight } \\
\text { Status }\end{array}$ & $\begin{array}{l}\text { CNV } \\
\text { Type }\end{array}$ & Cytoband & $\begin{array}{l}\text { Genome } \\
\text { Coordinate }\end{array}$ & Size & Origin & $\begin{array}{l}\text { Clinical } \\
\text { significance }\end{array}$ & RefSeq genes \\
\hline & & & & & & & & & & $\begin{array}{l}\text { EFTUD2, } \mathrm{PLCD3}, \text { CRHR1, } \\
\text { KANSL1 }\end{array}$ \\
\hline \multirow[t]{2}{*}{ P37 } & $8 y$ & $\mathrm{~F}$ & $\begin{array}{l}\text { BMI 22.3 } \\
\text { (97.4th) }\end{array}$ & Dup & 19p13.2 & $\begin{array}{l}12640509- \\
13231703\end{array}$ & 591194 & Unk & $\begin{array}{l}\text { 19p13.2 } \\
\text { duplication }\end{array}$ & MAST1, CALR, NFIX \\
\hline & & & & Dup & $9 p 22.1$ & $\begin{array}{l}19066513- \\
19497724\end{array}$ & 431211 & Unk & Uncertain & $\underline{\mathrm{PLIN2}}^{*}$ \\
\hline P38 & $18 y$ & $\mathrm{~F}$ & $\begin{array}{l}\text { BMl } 41.5 \\
\text { (99th) }\end{array}$ & Del & $19 p 13.12$ & $\begin{array}{l}14384925- \\
16034584\end{array}$ & 1649659 & $\begin{array}{l}\text { De } \\
\text { novo }\end{array}$ & $\begin{array}{l}\text { 19p13.12 } \\
\text { deletion }\end{array}$ & $\begin{array}{l}\text { CD97, DDX39A, PKN1, PTGER1, GIPC1, } \\
\text { CASP14, NOTCH3, CYP4F11 }\end{array}$ \\
\hline P39 & $8 y$ & $\mathrm{~F}$ & $\begin{array}{l}\text { Referred as } \\
\text { obese }\end{array}$ & Del & $22 q 11.21$ & $\begin{array}{l}18890162- \\
20311554\end{array}$ & 1421392 & $\begin{array}{l}\text { De } \\
\text { novo }\end{array}$ & 22q11.2 deletion & $\begin{array}{l}\text { PRODH, DGCR2, DGCR14, CDC45, } \\
\text { TBX1, * GNB1L, TXNRD2, COMT, * DGCR8, } \\
\text { ZDHHC8 }\end{array}$ \\
\hline P40 & $3 y$ & M & $\begin{array}{l}\text { BMI 34.7 } \\
\text { (99.9th) }\end{array}$ & Del & $22 q 11.21$ & $\begin{array}{l}18661758- \\
21684798\end{array}$ & 3023040 & $\begin{array}{l}\text { De } \\
\text { novo }\end{array}$ & 22q11.2 deletion & $\begin{array}{l}\text { PRODH, DGCR2, DGCR14, CDC45, } \\
\text { TBX1, * GNB1L, TXNRD2, COMT, * DGCR8, } \\
\text { ZDHHC8, PI4KA, SLC74A }\end{array}$ \\
\hline P41 & $9 y$ & M & $\begin{array}{l}\text { BMI } 32.5 \\
\text { (99.6th) }\end{array}$ & Del & $22 q 11.21$ & $\begin{array}{l}18661758- \\
21684798\end{array}$ & 3023040 & $\begin{array}{l}\text { De } \\
\text { novo }\end{array}$ & 22q11.2 deletion & $\begin{array}{l}\text { PRODH, DGCR2, DGCR14, CDC45, } \\
\text { TBX1, * GNB1L, TXNRD2, COMT, * DGCR8, } \\
\text { ZDHHC8, PI4KA, SLC74A }\end{array}$ \\
\hline P42 & $13 m$ & M & $\begin{array}{l}\text { Weight-for } \\
\text { Height }+1 \text { sd }\end{array}$ & Del & $22 q 11.21$ & $\begin{array}{l}18818429- \\
21661436\end{array}$ & 2843007 & Pat & 22q11.2 deletion & $\begin{array}{l}\text { PRODH, DGCR2, DGCR14, CDC45, } \\
\text { TBX1, * GNB1L, TXNRD2, COMT, * DGCR8, } \\
\text { ZDHHC8, PI4KA, SLC74A }\end{array}$ \\
\hline P43 & $18 y$ & M & $\begin{array}{l}\text { Referred as } \\
\text { obese }\end{array}$ & Dup & $22 q 11.21$ & $\begin{array}{l}18890162- \\
21464056\end{array}$ & 2573894 & Pat & $\begin{array}{l}22 q 11.2 \\
\text { duplication }\end{array}$ & $\begin{array}{l}\text { PRODH, DGCR2, DGCR14, CDC45, } \\
\text { TBX1, * GNB1L, TXNRD2, COMT, }{ }^{*} \text { DGCR8, } \\
\text { ZDHHC8, PI4KA, } \underline{\mathrm{SLC74}} \text {, }\end{array}$ \\
\hline P44 & $5 y$ & M & $\begin{array}{l}\text { BMI } 27.9 \\
\text { (99.9th) }\end{array}$ & Del & $\begin{array}{l}22 q 11.21 \\
q 11.23\end{array}$ & $\begin{array}{l}21759572- \\
23822925\end{array}$ & 2063353 & Unk & $\begin{array}{l}22 \mathrm{q} 11.2 \\
\text { deletion, distal }\end{array}$ & $H I C 2, \underline{M A P K 1}, *{ }^{*} G N A Z, B C R$ \\
\hline \multirow[t]{2}{*}{ P45 } & $7 y$ & $\mathrm{~F}$ & $\begin{array}{l}\text { BMI } 24.8 \\
\text { (98.9th) }\end{array}$ & Del & $\begin{array}{l}22 q 11.21 \\
q 11.22\end{array}$ & $\begin{array}{l}21468437- \\
22959609\end{array}$ & 1491172 & $\begin{array}{l}\text { Not } \\
\text { mat }\end{array}$ & $\begin{array}{l}22 \mathrm{q} 11.2 \\
\text { deletion, distal }\end{array}$ & HIC2, MAPK1* \\
\hline & & & & Dup & $3 p 26.3$ & $\begin{array}{l}857110- \\
1414719\end{array}$ & 557609 & $\begin{array}{l}\text { Not } \\
\text { mat }\end{array}$ & Uncertain & - \\
\hline P46 & $2 y$ & $\mathrm{~F}$ & $\begin{array}{l}\text { BMI } 17.7 \\
\text { (85th) }\end{array}$ & Del & $\begin{array}{l}22 \mathrm{q} 11.22 \\
\mathrm{q} 11.23\end{array}$ & $\begin{array}{l}23012069- \\
23648827\end{array}$ & 636758 & Mat & $\begin{array}{l}22 \mathrm{q} 11.2 \\
\text { deletion, distal }\end{array}$ & $G N A Z, B C R$ \\
\hline P47 & $15 y$ & M & $\begin{array}{l}\text { BMI 39.5 } \\
\text { (99.6th) }\end{array}$ & Del & $\begin{array}{l}22 \mathrm{q} 11.22 \\
\mathrm{q} 11.23\end{array}$ & $\begin{array}{l}23063178- \\
23696464\end{array}$ & 633286 & Unk & $\begin{array}{l}\text { 22q11.2 } \\
\text { deletion, distal }\end{array}$ & $G N A Z, B C R$ \\
\hline \multicolumn{11}{|c|}{ Other pathogenic imbalances } \\
\hline \multirow[t]{2}{*}{ P48 } & $2 y$ & M & $\begin{array}{l}\text { BMI } 24.4 \\
\text { (99.9th) }\end{array}$ & Del & $3 p 26.3$ & $\begin{array}{l}73603- \\
1273300\end{array}$ & 1199697 & $\begin{array}{l}\text { De } \\
\text { novo }\end{array}$ & $\begin{array}{l}\text { Unbalanced } \\
\text { translocation }\end{array}$ & $\mathrm{CHL} 1$ \\
\hline & & & & Dup & $\begin{array}{l}11 q 22.3 \\
q 25\end{array}$ & $\begin{array}{l}106251478- \\
134668665\end{array}$ & 28417187 & & & 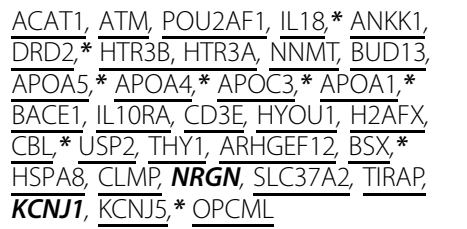 \\
\hline \multirow[t]{2}{*}{ P49 } & $14 y$ & $\mathrm{~F}$ & $\begin{array}{l}\text { Referred as } \\
\text { obese }\end{array}$ & Del & $3 p 24.1$ & $\begin{array}{l}28719852- \\
30169971\end{array}$ & 1450119 & Unk & $\begin{array}{l}\text { Complex } \\
\text { rearrangement }\end{array}$ & $\begin{array}{l}\text { LINC00693, RBMS3-AS3, RBMS3, RBMS3- } \\
\text { AS1 }\end{array}$ \\
\hline & & & & Dup & $\begin{array}{l}3 q 11.2 \\
q 13.31\end{array}$ & $\begin{array}{l}93558505- \\
115890384\end{array}$ & 22331879 & & & 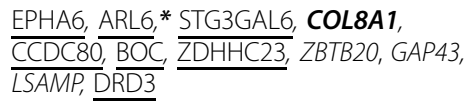 \\
\hline \multirow[t]{2}{*}{ P50 } & $10 y$ & M & $\begin{array}{l}\text { BMI } 30.4 \\
\text { (99.3th) }\end{array}$ & Del & $3 q 25.33$ & $\begin{array}{l}159252702- \\
160555217\end{array}$ & 1302515 & $\begin{array}{l}\text { De } \\
\text { novo }\end{array}$ & Uncertain & $\underline{\mathrm{IL} 12 \mathrm{~A}}$ \\
\hline & & & & Del & $\begin{array}{l}13 q 31.2 \\
q 32.1\end{array}$ & $\begin{array}{l}89522636- \\
95065310\end{array}$ & 5542674 & $\begin{array}{l}\text { De } \\
\text { novo }\end{array}$ & $\begin{array}{l}\text { Feingold } \\
\text { syndrome }\end{array}$ & MIR17HG, GPC5, ${ }^{*}$ GPC6 \\
\hline P51 & $14 y$ & $\mathrm{~F}$ & $\begin{array}{l}\text { BMI } 27.9 \\
\text { (95.8th) }\end{array}$ & Del & $\begin{array}{l}7 q 22.1 \\
q 22.3\end{array}$ & $\begin{array}{l}102358320- \\
105487655\end{array}$ & 3129335 & $\begin{array}{l}\text { De } \\
\text { novo }\end{array}$ & $\begin{array}{l}\text { Clinically } \\
\text { relevant }\end{array}$ & $\underline{\text { NAPEPLD, }}$ RELN, LHFPL3 \\
\hline
\end{tabular}


Table 3 Pathogenic copy number variations (PCNVs) detected in 279 patients with syndromic obesity (Continued)

\begin{tabular}{|c|c|c|c|c|c|c|c|c|c|c|}
\hline $\begin{array}{l}\text { Case } \\
\text { number }\end{array}$ & Age & Gender & $\begin{array}{l}\text { Weight } \\
\text { Status }\end{array}$ & $\begin{array}{l}\text { CNV } \\
\text { Type }\end{array}$ & Cytoband & $\begin{array}{l}\text { Genome } \\
\text { Coordinate }\end{array}$ & Size & Origin & $\begin{array}{l}\text { Clinical } \\
\text { significance }\end{array}$ & RefSeq genes \\
\hline \multirow[t]{2}{*}{ P52 } & $2 y$ & $F$ & $\begin{array}{l}\text { BMI 27.0 } \\
\text { (99.9th) }\end{array}$ & Del & $\begin{array}{l}\text { 10p15.3 } \\
\text { p14 }\end{array}$ & $\begin{array}{l}269695- \\
11579546\end{array}$ & 11309851 & $\begin{array}{l}\text { De } \\
\text { novo }\end{array}$ & $\begin{array}{l}\text { Unbalanced } \\
\text { translocation }\end{array}$ & 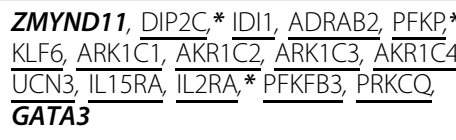 \\
\hline & & & & Dup & $6 q 27$ & $\begin{array}{l}169505179- \\
170694486\end{array}$ & 1189307 & & & WDR27 \\
\hline \multirow[t]{2}{*}{ P53 } & $5 m$ & $\mathrm{~F}$ & $\begin{array}{l}\text { Referred as } \\
\text { obese }\end{array}$ & Dup & $\begin{array}{l}\text { 10p15.3 } \\
\text { p12.31 }\end{array}$ & $\begin{array}{l}119794- \\
19509585\end{array}$ & 19389791 & $\begin{array}{l}\text { Not } \\
\text { mat }\end{array}$ & $\begin{array}{l}\text { Complex } \\
\text { rearrangement }\end{array}$ & 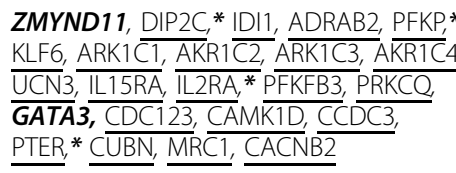 \\
\hline & & & & Dup & $\begin{array}{l}13 q 11 \\
q 12.3\end{array}$ & $\begin{array}{l}19440913- \\
31031907\end{array}$ & 11590994 & $\begin{array}{l}\text { Not } \\
\text { mat }\end{array}$ & & 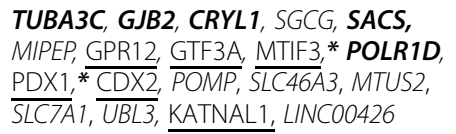 \\
\hline P54 & $9 y$ & $\mathrm{~F}$ & $\begin{array}{l}\text { BMI } 34.3 \\
\text { (99.7th) }\end{array}$ & Dup & $\begin{array}{l}10 q 26.11 \\
q 26.3\end{array}$ & $\begin{array}{l}120306959- \\
135434409\end{array}$ & 15127450 & $\begin{array}{l}\text { De } \\
\text { novo }\end{array}$ & $\begin{array}{l}\text { 10qter } \\
\text { duplication }\end{array}$ & 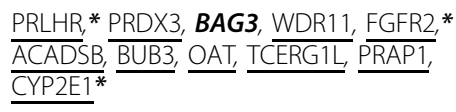 \\
\hline P55 & $7 y$ & $\mathrm{~F}$ & $\begin{array}{l}\text { BMI 23.6 } \\
\text { (98.8th) }\end{array}$ & Del & $\begin{array}{l}12 \mathrm{q} 15 \\
\mathrm{q} 21.1\end{array}$ & $\begin{array}{l}70555659- \\
73153191\end{array}$ & 2597532 & $\begin{array}{l}\text { De } \\
\text { novo }\end{array}$ & $\begin{array}{l}\text { Clinically } \\
\text { relevant }\end{array}$ & $\underline{\text { PTPRB, }}, \underline{\text { TSPAN8, }} *$ LGR5TPH2 \\
\hline P56 & $13 y$ & $\mathrm{~F}$ & $\begin{array}{l}\text { BMI } 36.8 \\
\text { (99.4th) }\end{array}$ & Dup & $\begin{array}{l}12 \mathrm{q} 21.32 \\
\mathrm{q} 23.1\end{array}$ & $\begin{array}{l}88684581- \\
101464859\end{array}$ & 12780278 & $\begin{array}{l}\text { De } \\
\text { novo }\end{array}$ & $\begin{array}{l}\text { Insertional } \\
\text { translocation }\end{array}$ & $\frac{\mathrm{KITLG}}{\underline{\mathrm{NR} 1 \mathrm{H}} 4^{*}}, \underline{\text { ATP2B1 }}, \underline{\text { SOCS2}}, \underline{\text { LTA4H }}, \underline{\text { RMST, }}$ \\
\hline P57 & $15 y$ & M & $\begin{array}{l}\text { BMl } 43.1 \\
\text { (99.7th) }\end{array}$ & Del & $\begin{array}{l}13 q 33.2 \\
q 34\end{array}$ & $\begin{array}{l}106648660- \\
115105655\end{array}$ & 8456995 & $\begin{array}{l}\text { Not } \\
\text { mat }\end{array}$ & 13qter deletion & 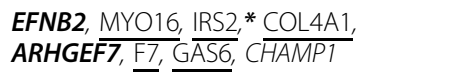 \\
\hline P58 & $4 y$ & M & $\begin{array}{l}\text { BMI 22.0 } \\
\text { (99.9th) }\end{array}$ & Dup & $14 q 11.2$ & $\begin{array}{l}21244696- \\
22250879\end{array}$ & 1006183 & $\begin{array}{l}\text { De } \\
\text { novo }\end{array}$ & $\begin{array}{l}14 q 11.2 \\
\text { microduplication }\end{array}$ & SUPT16H, CHD8 \\
\hline \multirow[t]{2}{*}{ P59 } & $16 y$ & $\mathrm{~F}$ & $\begin{array}{l}\text { BMI } 38.5 \\
\text { (99th) }\end{array}$ & Del & $14 q 12$ & $\begin{array}{l}29781404- \\
30552936\end{array}$ & 771532 & $\begin{array}{l}\text { De } \\
\text { novo }\end{array}$ & $\begin{array}{l}14 \mathrm{q} 12 \text { deletion, } \\
\text { non-critical }\end{array}$ & $\underline{\mathrm{PRKD}}^{*}$ \\
\hline & & & & Dup & $4 p 16.1$ & $\begin{array}{l}10068064- \\
10529023\end{array}$ & 460959 & Mat & Likely benign & WDR1 \\
\hline P60 & $7 y$ & $\mathrm{~F}$ & $\begin{array}{l}\text { BMI 24.8 } \\
\text { (99.3th) }\end{array}$ & Del & $\begin{array}{l}\text { Xp22.12 } \\
\text { p22.13 }\end{array}$ & $\begin{array}{l}18214020- \\
19833634\end{array}$ & 1619614 & Unk & $\begin{array}{l}\text { Rett syndrome- } \\
\text { like }\end{array}$ & CDKL5, RS1, $\underline{\mathrm{PHKA} 2,} \underline{\mathrm{PDHA} 1}, *{ }^{\mathrm{SH} 3 \mathrm{KBP} 1}$ \\
\hline \multirow[t]{2}{*}{ P61 } & $14 y$ & M & $\begin{array}{l}\text { BMI } 37.0 \\
\text { (99.5th) }\end{array}$ & Dup & Xp22.3 & $\begin{array}{l}75943- \\
2685605\end{array}$ & 2609662 & $\begin{array}{l}\text { De } \\
\text { novo }\end{array}$ & $\begin{array}{l}\text { Complex } \\
\text { rearrangement }\end{array}$ & SHOX, ASMTL, ASMT \\
\hline & & & & Dup & $\begin{array}{l}X q 21.31 \\
q 21.32\end{array}$ & $\begin{array}{l}88489522- \\
92357353\end{array}$ & 3867831 & & & TGIF2 LX, PABPC5-AS1, PABPC5, PCDH11X \\
\hline
\end{tabular}

Abbreviations: $M$ male, $F$ female, Del deletion, Dup duplication, y years, $m$ months, $B M I$ body mass index, SD standard deviation, unk unknown, mat maternally inherited, pat paternally inherited, not mat not maternally inherited; Genes in bold were listed in the CNV morbidity map of IDDs [34]. Underlined genes were retrieved from the Text-mined Hypertension, Obesity and Diabetes candidate gene database (T-HOD), the Human Genome Epidemiology (HUGE) Phenopedia, and from a list of obesity candidate genes curated from the literature [33]. Genes found at the intersection of at least two gene sets are highlighted (asterisks). Patients $6-12,14,15,18,23,28,30-33,35,40-43,46-48,51,52,55,56,58-60$ ) have been published previously as separate studies [36-38]

PCNVs in children and adolescents did not differ significantly between gender and age groups, and obesity severity (Fig. 7). Nevertheless, females had a higher detection rate of PCNVs in comparison to males (27\% females and $20 \%$ males; overall), with the highest differences (35\% females and $11 \%$ males) found in the younger age group (2-9 years) with less severe grades of obesity (BMI $<1.2 \times 95$ th percentile). Whilst no single phenotypic feature could be investigated for association with PCNV risk, due to the absence of comprehensively phenotyping of patients, phenotypephenotype correlation analysis between cases with and without PCNVs identified 12 pairs of phenotypes that were significantly associated with the presence of PCNVs and combining hypotonia, language impairments, abnormalities of the external genitalia, and eye/vision problems at its core. Of note, patients in our cohort were almost 10 times more likely to manifest macrocephaly as compared to microcephaly. Even though $33 \%$ of macrocephalic patients displayed PCNVs, macrocephaly did not associate with the presence of PCNVs.

In the current study, we have identified known genomic imbalance disorders in 47 patients, and, of them, 35 patients (13\%) carry overlapping and recurrent CNVs (Table 3; Fig. 3). In our cohort, imbalances that are known to be causal for syndromic obesity were observed in 23 patients $(8.2 \%)$. The most commonly identified 


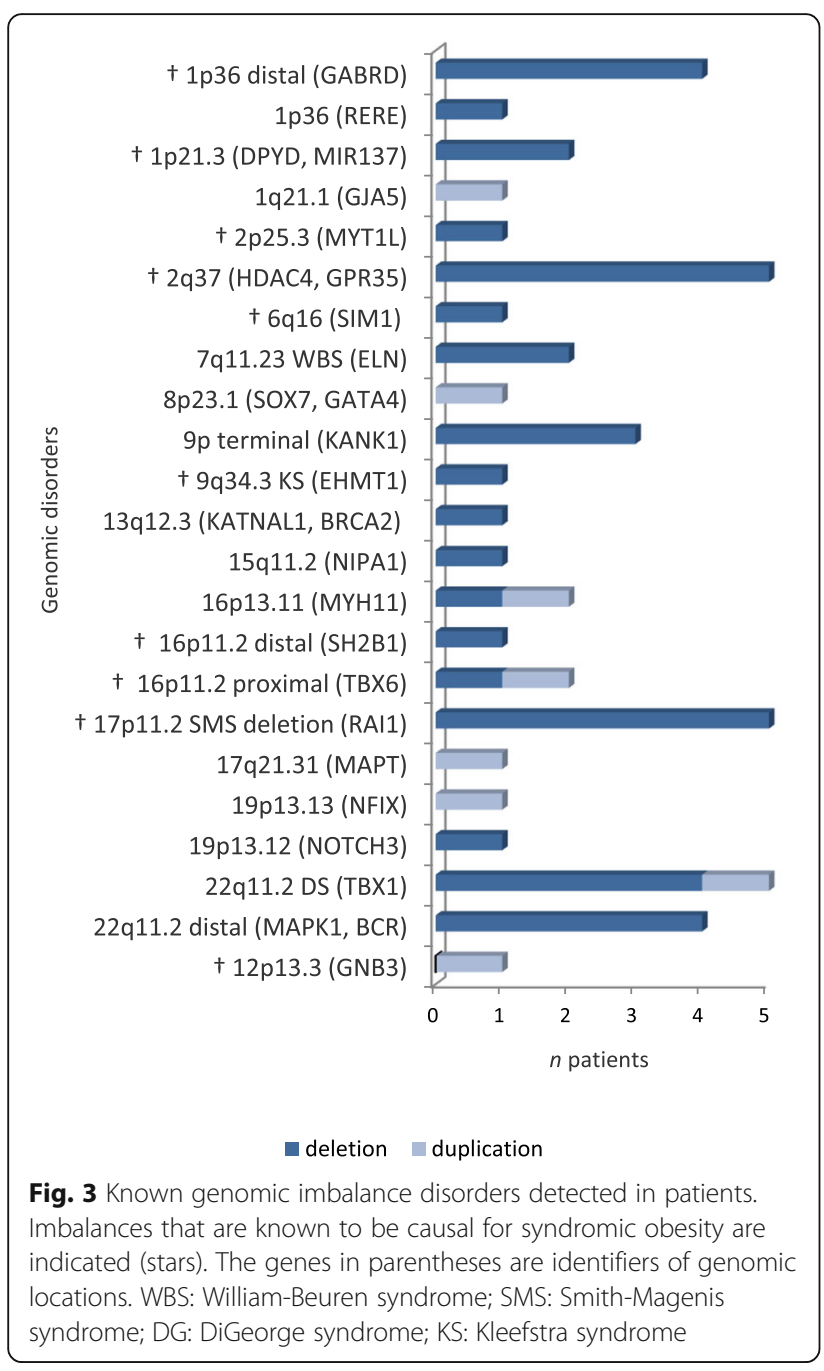

syndromic forms of obesity were deletions of the chromosomal regions 1p36, 2q37 and 17p11.2, which collectively represented $5.4 \%$ of all cases, followed by microdeletions of the $1 \mathrm{p} 21.3$ region ( 2 cases). In 6 other syndromic obesity loci $(2 \mathrm{p} 25.3,6 \mathrm{q} 16,9 \mathrm{q} 34,16 \mathrm{p} 11.2$ proximal and distal, 12p13.31), CNVs were found only in one unrelated individual. The identification of CNVs overlapping loci previously shown to be involved in syndromic obesity further implicates them as risk factors for obesity. As previously mentioned, SH2B1, SIM1, PTBP2, PRLH, CAPN10, ACP1, TMEM18, EHMT1, and GNB3 are relevant candidate and known genes for obesity within these regions (Fig. 6), and POU3F2 [20], HDAC4 [50], MYT1L [52], and RAI1 [53] were also candidate genes identified in these loci. Moreover, our gene prioritization analysis identified 20 new genes of interest to obesity overlapping these CNVs, among which we highlight the potential importance of TAS1R3, encoding a taste receptor differentially expressed in obese mice
[54]. This gene maps within the common deleted region of patients with distal $1 \mathrm{p} 36$ deletion.

In addition to the above, we identified 4 patients with recurrent deletions at the 22q11.2 DiGeorge syndrome (DS) region (we also found a patient with duplication of the same region), 4 patients with distal 22q11.2 recurrent deletions, and 3 patients with overlapping deletions at $9 p$ terminal. As these CNVs arise in more than 2 unrelated individuals, we implicate them as novel loci with a potential role in obesity susceptibility. A link between the $22 q 11.2$ region with obesity is also supported by previous works showing that 22q11.2DS deletion carriers have increased rates of obesity [55-57], as well as reports of patients presenting childhood obesity with hyperphagia $[58,59]$. Overweight and obesity (with or without hyperphagia) have also been described in a number of patients with distal 22q11.2 deletions [60-63]. We identified 3 genes at 22q11.2 (TBX1, COMT and MAPK1) that could confer susceptibility to obesity (Fig. 6). Although obesity is not a reported feature of deletion 9p syndrome, weight $\geq$ 90th percentile at birth or in childhood was documented in 4 of a series of 10 patients with distal deletions of $9 p$ [64], further emphasizing the potential importance of this region. Additionally, we recently detected a deletion at 9 p24.3p24.2 in one further patient with syndromic obesity using multiplex ligation-probe amplification (unpublished data from our laboratory). The VLDLR, IL33 and PTPRD genes were identified as the most interesting genes for obesity-susceptibility within 9 p24 (Fig. 6). Furthermore, we detected 2 patients with Williams-Beuren syndrome (WBS) 7q11.23 deletions. This region was already shown to be associated with several endocrine and metabolic problems including hypothyroidism, hypercalcemia, obesity and diabetes $[65,66]$. Two genes related to obesity, STX1A and $R F C 2$, map to this $\mathrm{CNV}$ interval (Fig. 6).

In this study we discovered recurrent CNVs at 1q21.1 and 16p13.1, which are known predisposing factors to IDDs reported sometimes in patients exhibiting obesity [67, 68]. Moreover, CNVs at these loci were also documented in a cohort study of syndromic obesity [22]. We also discovered other CNVs overlapping loci involved in syndromic obesity cases from the literature and DECIPHER. For instance, patient 24 carry a deletion overlapping the critical region of the 13q12.3 microdeletion syndrome described by Bartholdi et al. in 3 unrelated patients, two of whom with obesity [69]. This deletion was further associated with obesity in a patient from DECHIPER (case 282,282). Five genes map to the common $\mathrm{CNV}$ interval (Fig. 4a), among them $A L O X 5 A P$ whose expression was linked to obesity and insulin resistance [70]. Likewise, patient 38 carry a deletion at 19 p13.12 partially overlapping with those reported in 3 patients from literature, one of them with obesity [71]. We found 2 other patients in DECIPHER with deletions at this 


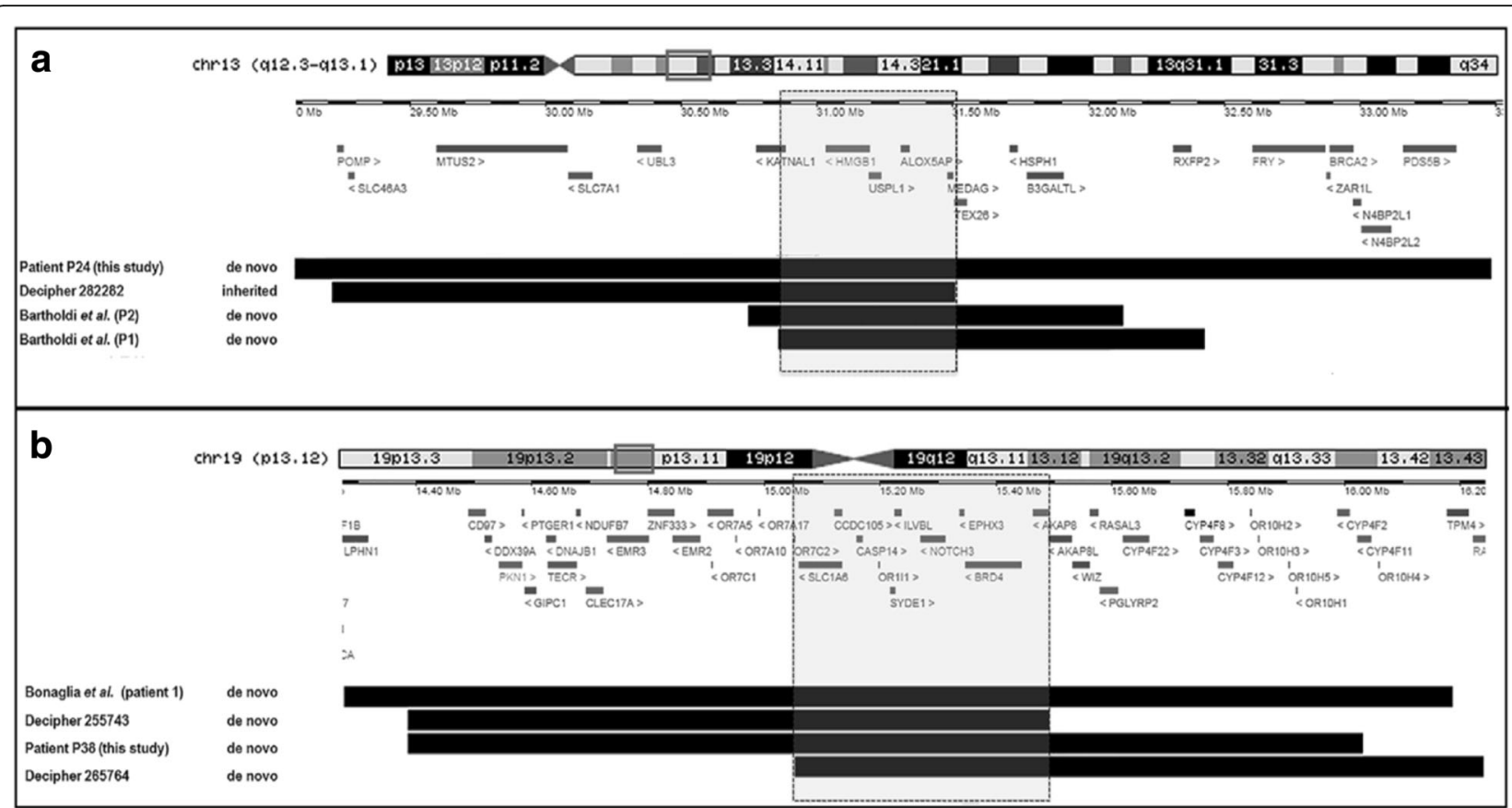

Fig. 4 Schematic alignment using the UCSC Genome Browser custom track tools (hg19) showing the regions of overlap between the deleted segments detected in (A) patient 24 (this study), DECIPHER case 282,282, and patients 1 and 2 of Bartholdi's study [69]; (B) patient 38 (this study), DECIPHER case 255,743 and 265,764, and patient 1 of Bonaglia's study [71]. The inheritance of the CNVs and the protein-coding genes located within them are shown. The inner light gray boxes show the smallest regions of overlapping deletions

locus in addition to obesity (cases 255,743 and 265,764). These cases share a 440-kb SRO encompassing 9 genes, including NOTCH3 (Fig. 4b). The Notch signaling has recently emerged as a key player in regulating metabolism [72]. We also identified a case of 19p13.2 duplication involving the NFIX gene associated with a Sotos syndromelike phenotype [73]. This CNV was associated with a 430kb 9p22.1 duplication that encompassed the entire PLIN2 gene, which is involved in the control of energy balance [74]. Notably, CNV in this gene has previously been identified in a patient with syndromic obesity [22].

Other than CNVs overlapping known genomic disorders loci, 14 patients had other chromosomal defects that are known to be clinically relevant, among which 4 overlapped with loci previously implicated in obesity. The distal portion of the 3q11.2q13.31 duplication in patient 49 (Fig. 5a) partially overlaps with a $2.76-\mathrm{Mb} 3 \mathrm{q} 13.31$ duplication found in 2 brothers with syndromic obesity [22], and with a 9.8-Mb 3q13.13q13.32 duplication reported in association with obesity from DECIPHER (case 314,391). The common region of overlap involves 5 genes and among them ZBTB2O is implicated in Primrose syndrome associated with several endocrine features and obesity (OMIM \#259050). The large 13q11q12.3 duplication in our patient 53 (Fig. 5b) overlaps with a $1.2-\mathrm{Mb}$ 13q12.12 duplication also found in our patient 19 and with another 2-Mb 13q12.11q12.12 duplication detected among cases with moderate and extreme obesity [75]. This region involves the gene SGCG with expression in adipose tissues and associated with type 2 diabetes [76]. Patient 57 carry an $8.5-\mathrm{Mb} 13 \mathrm{q} 33.2 \mathrm{q} 34$ deletion encompassing the ID gene CHAMP1 (Fig. 5c), which partially overlaps with deletions at 13q34 found in 6 other patients with syndromic obesity reported by Vuillaume et al. [22] and Reinstein et al. [21]. Of interest, the deleted region in each of the 7 cases overlaps the obesity-associated gene GAS6 [77]. The 14q11.2 microduplication found in our patient 58 (Fig. 5d), including the SUPT16H and CHD8 genes, was also identified in one patient with syndromic obesity reported by Vuillaume et al. [16]. No candidate genes for obesity were associated with this locus. Although there is one case of 14q11.2 deletion that was reported with severe obesity, it included a large more proximal segment of $14 \mathrm{q} 11.2$, which contains a strong obesity candidate gene [14].

Finally, in a total of 51 patients the CNVs were classified as potentially pathogenic (2.1\%), VUS $(4.7 \%)$ or likely benign variants $(11.5 \%)$. Overall, a number of interesting genes that could play a role in obesity susceptibility have been identified within these CNVs (e.g. ASTN2, APOA2, PARK2, LINGO2, PLCB1, PTEN, and CIDEA). More importantly, we identified a new and de novo $340-\mathrm{kb}$ 16p13.2 duplication that encompasses the entire USP7 gene. Although its pathogenicity is not certain, since 


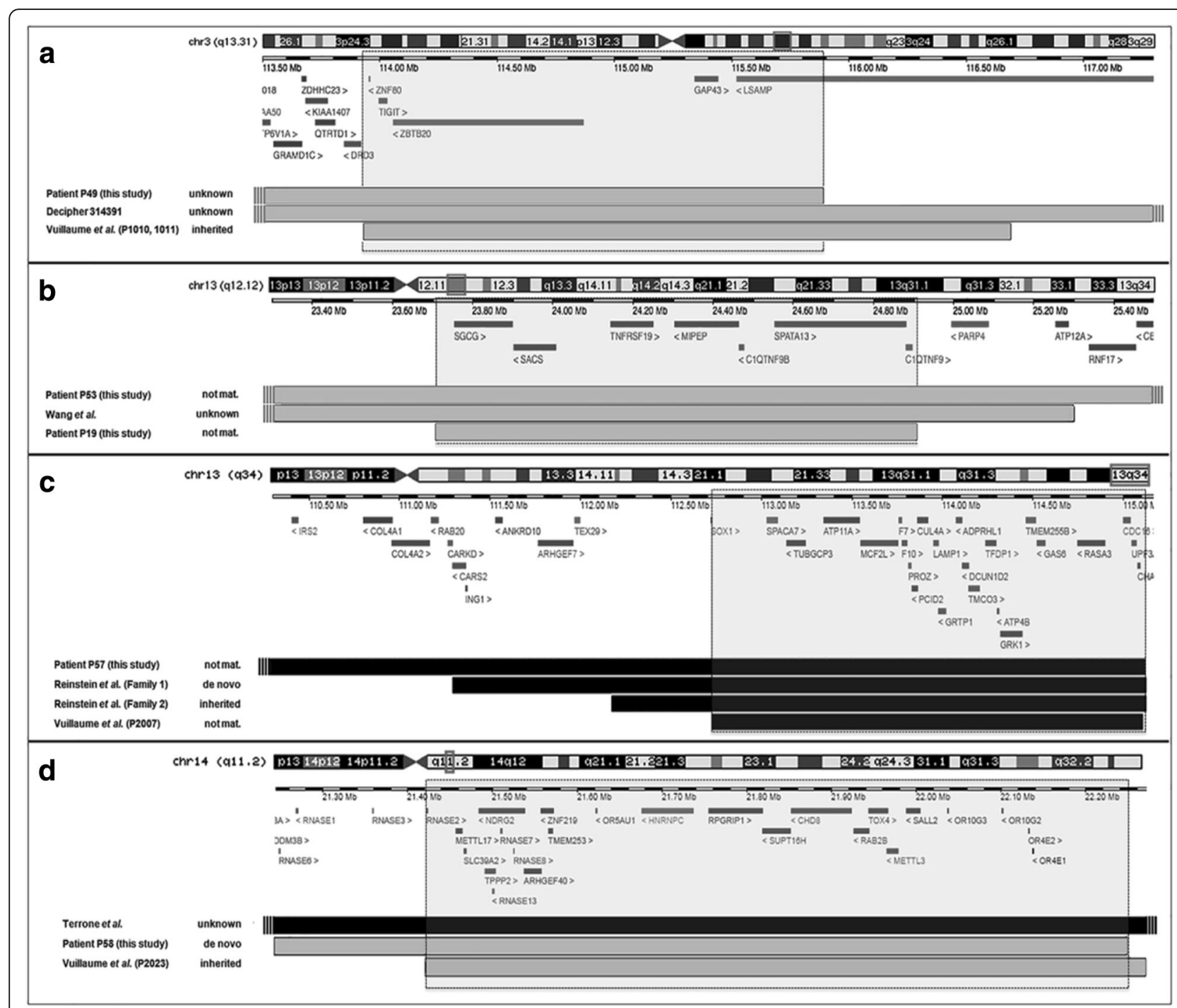

Fig. 5 Schematic alignment using the UCSC Genome Browser custom track tools (hg19) showing the regions of overlap between deleted (black) and duplicated (grey) segments detected in (a) patient 19 (this study), DECIPHER case 314,391 and brothers P1010 and P1011 of Vuillaume's study [22]; (b) patients 19 and 53 (this study) and one individual described by Wang et al. [75]; (c) patient 57 (this study), case P2007 of Vuillaume's study [22] and families 1 and 2 of Reinstein's study [21]; (d) patient 58 (this study), case P2023 of Vuillaume's study [22], and one patient described by Terrone et al. [14]. Vertical lines depict breakpoints that extended beyond the regions indicated here. The inheritance of the CNVs and the protein-coding genes located within them are shown. The inner light gray boxes show the smallest regions of overlap

similar duplications have not been reported in the literature, there is one reported patient with larger duplication at the USP7 locus presenting with severe early-onset obesity and hyperphagia [78]. Of note, USP7 has been identified as an integral component of MAGEL2 and TRIM27 ubiquitin ligase complex, which plays an important role in hypothalamic function [79]. Moreover, deletion or mutation of USP7 has been shown to result in a neurodevelopmental disorder with overlapping symptoms to Schaaf-Yang syndrome (OMIM \#615547), caused by mutations of MAGEL2 [78]. There are 5 de novo duplication events overlapping USP7 (400-kb to $1.2-\mathrm{Mb})$ reported in DECIPHER with no additional changes detected. These included 3 patients (cases 269,501, 281,449 and 258,037) with delayed speech and language development as common features and 2 patients (cases 254,000 and 267,094 ) with no phenotypic description. One of the limitations of our study is that additional independent risk factors were not considered, including unidentified genetic factors and those being epigenetic, environmental, or stochastic in origin. Future investigations of genes within disease-specific CNVs detected in the present cohort are also needed. Future directions will involve whole exome sequencing (WES) in patients 


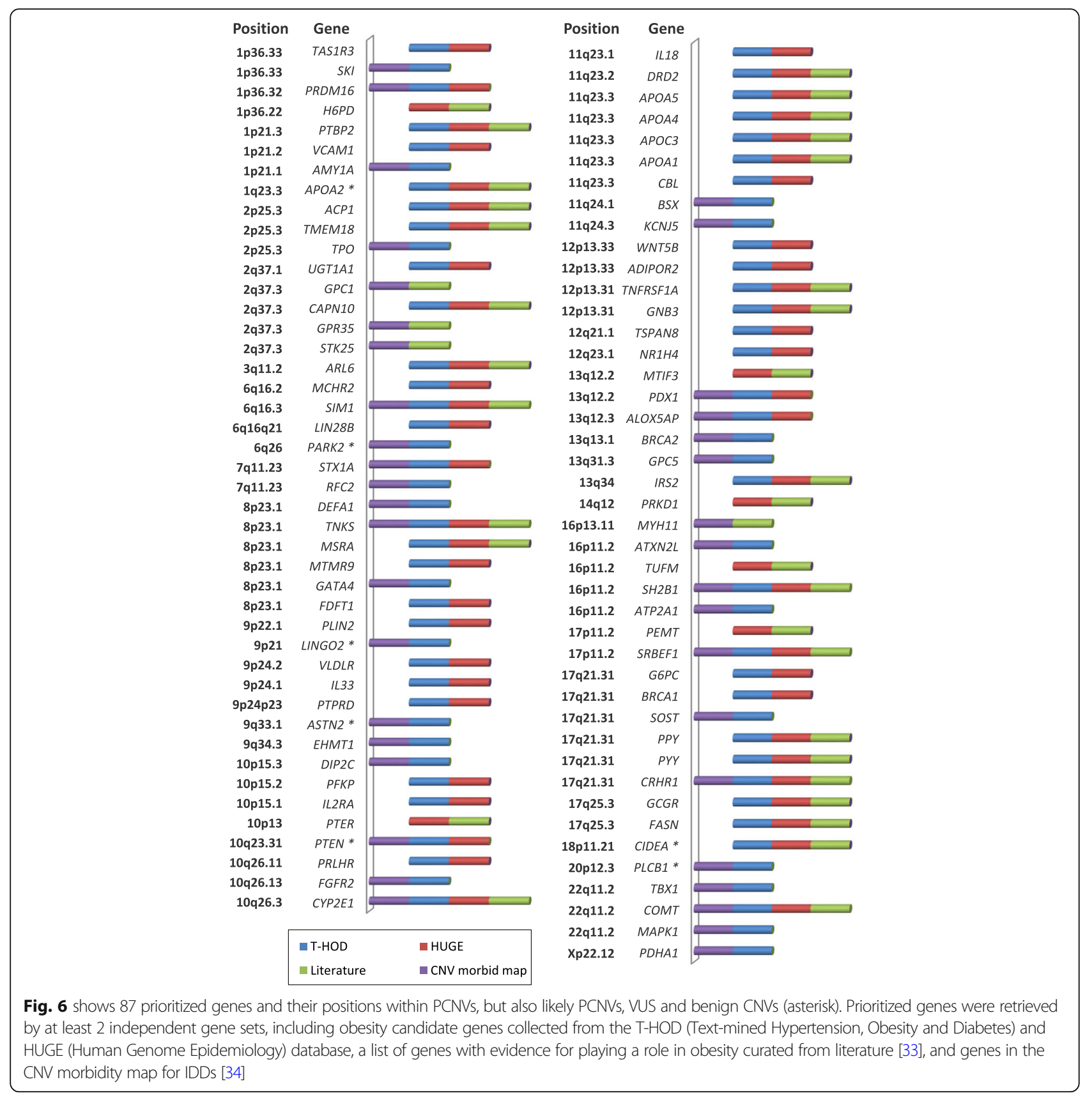

that did not reach a diagnosis to estimate the contribution of single gene mutations in the genetic causation of syndromic obesity. This will allow isolate genes that cause or may affect susceptibility to obesity in humans, advancing our understanding of the molecular mechanisms involved in body weight regulation and provide clues for therapeutic intervention in obesity.

\section{Conclusion}

Understanding the genetics of obesity has proven difficult. Although it is likely that not all of the PCNVs detected in the current study are directly causative of obesity, we found that $23 / 279$ (8.2\%) of our patients carried rare CNVs at 10 loci already known to increase the risk of obesity. We identified 3 patients with overlapping deletions at $9 \mathrm{p}$ terminal, 4 patients with deletions of 22q11.2DS and 4 patients with deletions at distal 22q11.2, which thus emerge as new putative obesity-susceptibility loci. In addition, we found that CNVs in at least 6 other cases overlapped with loci previously implicated in syndromic obesity, including a new patient with deletion at chromosome 13q34. This 

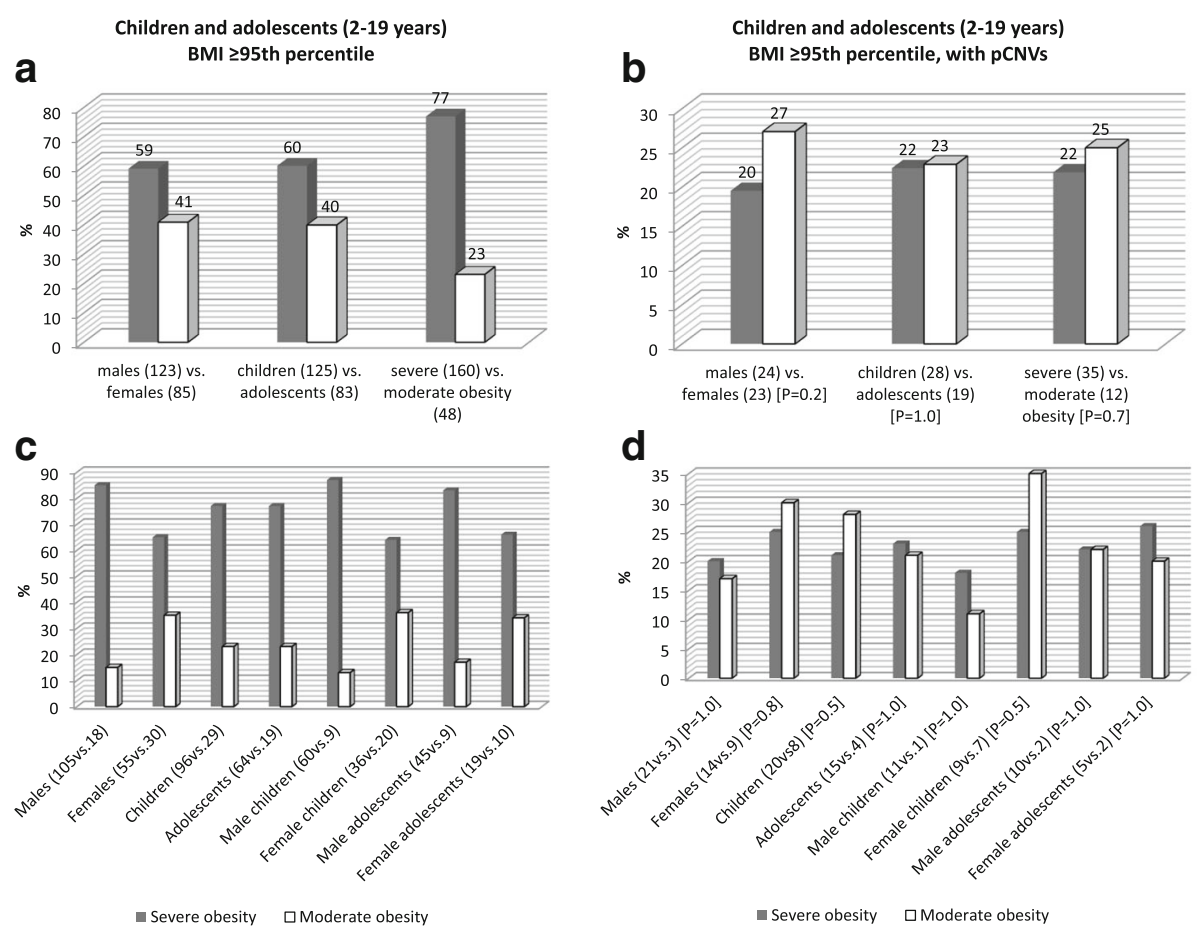

Fig. 7 (A) Frequencies of children and adolescents in the whole cohort with BMI $\geq 95$ th percentile stratified by gender (males and females), age groups (2-9 years and 10-19 years), and level of obesity (moderate and severe). (B) Sex- and age-related differences of children and adolescents in the whole cohort by level of obesity. (C, D) same comparisons in children and adolescents with PCNVs. The numbers in parentheses indicate the total number of patients in each group. Definition of moderate and severe obesity was based on the BMI below or above $120 \%$ of the 95 th percentile. $P$-values for the Fisher's exact test are shown

locus is particularly interesting because our new case brings to 7 the number of patients in whom such defects have been described in association with obesity. Overall, we found CNVs that further implicate genes previously associated with obesity such as PTBP2, TMEM18, MYT1L, POU3F2, SIM1, SH2B1 and GNB3, and also identified other potentially relevant candidate genes including TAS1R3, ALOX5AP, and GAS6. Our study highlights the significant value of chromosomal microarrays in providing not only a genetic diagnosis for syndromic causes of obesity but in uncovering genes relevant to human obesity.

\section{Additional files}

Additional file 1: Table S1. Full description of clinical findings in patients enrolled in this study. (XLSX $71 \mathrm{~kb}$ )

Additional file 2: Table S2. Frequencies of phenotypes pairs segregating together in patients with and without pCNVs. (XLSX 18 kb)

Additional file 3: Table S3. Likely PCNVs, VUS and likely benign variants detected in patients with syndromic obesity (XLSX $19 \mathrm{~kb}$ )

Additional file 4: Table S4. Evaluation of the level of obesity in 208 children and adolescents with BMI $\geq 95$ th percentile. (PDF $81 \mathrm{~kb}$ )

Additional file 5: Table S5. Comparison of the pCNVs rates for children and adolescents with BMI at or above the 95th percentile by age, sex and level of obesity. (PDF $81 \mathrm{~kb}$ )

\section{Abbreviations}

ACMG: American college of medical genetics; ASD: Autism spectrum disorder; BBS: Bardet-Biedl Syndrome; BMI: Body mass index; BP: Breakpoint; CDC: Centers for disease control and prevention; ClinGen: Clinical genome resource; CMA: Chromosomal microarray analysis; CNV: Copy number variant; $\mathrm{COH1}$ : Cohen syndrome; DECIPHER: DatabasE of genomiC varlation and phenotype in humans using ensembl resources; DG: DiGeorge syndrome; DGV: Database of genomic variants; HUGE: Human genome epidemiology; ID: Intellectual disability; IDD: Intellectual and developmental disabilities; ISCA: International standards for cytogenomic arrays; KS: Kleefstra syndrome; OFC: Occipitofrontal circumference; OMIM: Online mendelian inheritance in man; PCNV: Pathogenic copy number variant; PWS: Prader-Willi syndrome; SD: Standard deviation; SMS: Smith-Magenis syndrome; SRO: Smallest region of overlap; T-HOD: Text-mined hypertension, obesity and diabetes: VUS: Variants of uncertain significance; WBS: Williams-Beuren syndrome; WES: Whole-Exome sequencing

\section{Acknowledgements}

We thank the patients and their families for participating in our research studies and other clinicians for referral of patients. This study makes use of data generated by the DECIPHER community. A full list of centres who contributed to the generation of the data is available from http://decipher.sanger.ac.uk and via email from decipher@sanger.ac.uk. Funding for the project was provided by the Wellcome Trust.

\section{Funding}

This study was supported by The State of São Paulo Research Foundation, FAPESP (09/52523-1 to C.S.D.), The Centers for Research, Innovation and Diffusion, CEPID-FAPESP (1998/14254-2), and The National Council for Scientific and Technological Development, CNPq (304381/2007-1 to C.P.K.). 


\section{Availability of data and materials}

All data generated or analyzed during this study are included in this published article (and supplementary information file). Detailed clinical information of the patients reported in this paper are however available from the corresponding author on reasonable request.

\section{Authors' contributions}

C.S.D. and C.P.K. designed the study. C.S.D. performed the experiments, analyzed the data and wrote the manuscript. P.A.O. conducted the statistical analyses. A.B.A.P., C.M.L., C.A.K, D.R.B., F.K, and L.G.A. are the main referring clinicians and performed the clinical assessment and physical examination of patients. M.C.V. performed the methylation analysis of the PWS chromosome region. C.S.D. collected anthropometric measurements and phenotypic data with contribution from C.I.E.C. All authors read and approved the final manuscript.

\section{Ethics approval and consent to participate}

Informed consent was obtained from all patients' parents or quardians. Ethical approval of the study protocol was obtained from the Human Research Ethics Committee of the Institute of Biosciences, USP.

\section{Consent for publication}

All authors commented on and approved the manuscript.

\section{Competing interests}

The authors declare that they have no competing interests.

\section{Publisher's Note}

Springer Nature remains neutral with regard to jurisdictional claims in published maps and institutional affiliations.

\section{Author details}

${ }^{1}$ Human Genome and Stem Cell Research Center (HUG-CELL), Department of Genetics and Evolutionary Biology, Institute of Biosciences, University of Sao Paulo, Rua do Matao no 277, Cidade Universitaria-Butanta, Sao Paulo, SP 05508-090, Brazil. ${ }^{2}$ Department of Morphology and Genetics, Paulista School of Medicine, Federal University of Sao Paulo (UNIFESP), Sao Paulo, SP, Brazil. ${ }^{3}$ Neurogenetics Unit, Clinics Hospital of Ribeirao Preto, Faculty of Medicine, University of Sao Paulo, FMRP-USP, Ribeirao Preto, SP, Brazil. ${ }^{4}$ Genetic Unit, Children's Institute, Faculty of Medicine, University of Sao Paulo, FMUSP, Sao Paulo, SP, Brazil. ${ }^{5}$ Department of Neurology, Faculty of Medicine, University of Sao Paulo, FMUSP, Sao Paulo, SP, Brazil.

\section{Received: 12 October 2017 Accepted: 22 January 2018}

\section{Published online: 05 February 2018}

\section{References}

1. D'Angelo CS, Koiffmann CP. Copy Number variants in obesity-related syndromes: review and perspectives on novel molecular approaches. Obes. 2012;2012:845480

2. Huvenne $H$, Dubern $B$, Clément $K$, Poitou C. Rare genetic forms of obesity: clinical approach and current treatments in 2016. Obes Facts. 2016;9(3):158-73.

3. Pigeyre M, Yazdi FT, Kaur Y, Meyre D. Recent progress in genetics, epigenetics and metagenomics unveils the pathophysiology of human obesity. Clin Sci (Lond). 2016;130(12):943-86.

4. Davidsson J, Jahnke K, Forsgren M, Collin A, Soller M. Dup(19)(q12q13.2): array-based genotype-phenotype correlation of a new possibly obesityrelated syndrome. Obesity (Silver Spring). 2010;18(3):580-7.

5. Wentzel C, Lynch SA, Stattin EL, Sharkey FH, Annerén G, Thuresson AC. Interstitial deletions at 6q14.1-q15 associated with obesity, developmental delay and a distinct clinical phenotype. Mol Syndromol. 2010;1:75-81.

6. Oexle K, Hempel M, Jauch A, Meitinger T, Rivera-Brugués N, StengelRutkowski S, Strom T. 3.7 Mb tandem microduplication in chromosome 5p13.1-p13.2 associated with developmental delay, macrocephaly, obesity, and lymphedema. Further characterization of the dup(5p13) syndrome. Eur J Med Genet. 2011;54(3):225-30.

7. Halgren C, Bache I, Bak M, Myatt MW, Anderson CM, Brøndum-Nielsen K, Tommerup N. Haploinsufficiency of CELF4 at 18q12.2 is associated with developmental and behavioral disorders, seizures, eye manifestations, and obesity. Eur J Hum Genet. 2012;20(12):1315-9.

8. Vergult S, Dauber A, Delle Chiaie B, Van Oudenhove E, Simon M, Rihani A et al. 17q24.2 microdeletions: a new syndromal entity with intellectual disability, truncal obesity, mood swings and hallucinations. Eur J Hum Genet. 2012:20:534-9.

9. Shichiji M, Ito Y, Shimojima K, Nakamu H, Oguni H, Osawa M, Yamamoto T. A cryptic microdeletion including MBD5 occurring within the breakpoint of a reciprocal translocation between chromosomes 2 and 5 in a patient with developmental delay and obesity. Am J Med Genet A. 2013;161A(4):850-5

10. Bonaglia MC, Giorda R, Zanini S. A new patient with a terminal de novo 2p25.3 deletion of $1.9 \mathrm{Mb}$ associated with early-onset of obesity, intellectual disabilities and hyperkinetic disorder. Mol Cytogenet. 2014;7:53.

11. Courage C, Houge G, Gallati S, Schjelderup J, Rieubland C. 15q26.1 microdeletion encompaasing only CHD2 and RGMA in two adults with moderate intellectual disability, epilepsy and truncal obesity. Eur J Med Genet. 2014;57:520-3.

12. Doco-Fenzy M, Leroy C, Schneider A, Petit F, Delrue MA, Andrieux J, et al. Early-onset obesity and paternal 2pter deletion encompassing the ACP1, TMEM18, and MYT1L genes. Eur J Hum Genet. 2014;22:471-9.

13. Kuroda Y, Ohashi I, Tominaga M, Saito T, Nagai J, Ida K, et al. De novo duplication of 17p13.1-p13.2 in a patient with intellectual disability and obesity. Am J Med Genet A. 2014;164A(6):1550-4.

14. Terrone G, Cappuccio G, Genesio R, Esposito A, Fiorentino V, Riccitelli M, et al. A case of $14 q 11.2$ microdeletion with autistic features, severe obesity and facial dysmorphisms suggestive of wolf-Hirschhorn syndrome. Am J Med Genet A. 2014;164A(1):190-3.

15. Desch L, Marle N, Mosca-Boidron A-L, Faivre L, Eliade M, Payet M, et al. 6q16.3q23.3 duplication associated with Prader-Willi-like syndrome. Mol Cytogenet. 2015;8:42.

16. Biamino E, Di Gregorio E, Belligni EF, Keller R, Riberi E, Gandione M, et al. A novel 3q29 deletion associated with autism, intellectual disability, psychiatric disorders, and obesity. Am J Med Genet B. 2016;171B:290-9.

17. Goldlust IS, Hermetz KE, Catalano LM, Barfield RT, Cozad R, Wynn G, et al. Mouse model implicates GNB3 duplication in a childhood obesity syndrome. Proc Natl Acad Sci U S A. 2013;110:14990-4

18. Carter MT, Nikkel SM, Fernandez BA, Marshall CR, Noor A, Lionel AC, et al. Hemizygous deletions on chromosome 1p21.3 involving the DPYD gene in individuals with autism spectrum disorder. Clin Genet. 2011;80(5):435-43.

19. Willemsen $M H$, Valls A, Kirkels LA, Mastebroek M, Loohuis N, Kos A, et al. Chromosome 1p21.3 microdeletions comprising DPYD and MIR137 are associated with intellectual disability. J Med Genet. 2011;48:810-8.

20. Kasher PR, Schertz KE, Thomas M, Jackson A, Annunziata S, Ballesta-Martinez MJ, et al. Small 6q16.1 deletions encompassing POU3F2Cause susceptibility to obesity and variable developmental delay with intellectual disability. Am J Hum Genet. 2016;98(2):363-72.

21. Reinstein $E$, Liberman M, Feingold-Zadok M, Tenne T, Graham JM Jr. Terminal microdeletions of $13 q 34$ chromosome region in patients with intellectual disability: delineation of an emerging new microdeletion syndrome. Mol Genet Metab. 2016;118(1):60-3.

22. Vuillaume ML, Naudion S, Banneau G, Diene G, Cartault A, Cailley D, et al. New candidate loci identified by array-CGH in a cohort of 100 children presenting with syndromic obesity. Am J Med Genet A. 2014;164A:1965-75.

23. Kuczmarski RJ, Ogden CL, Grummer-Strawn LM, Flegal KM, Guo SS, Wei R, et al. CDC growth charts: United States. Adv Data. 2000;314:1-27.

24. Flegal KM, Wei $\mathrm{R}$, Ogden $\mathrm{CL}$, Freedman DS, Johnson $\mathrm{CL}$, Curtin LR. Characterizing extreme values of body mass index-for-age by using the 2000 Centers for Disease Control and Prevention growth charts. Am J Clin Nutr. 2009;90(5):1314-20.

25. Gulati AK, Kaplan DW, Daniels SR. Clinical tracking of severely obese children: a new growth chart. Pediatrics. 2012;130(6):1136-40.

26. Kearney HM, Thorland EC, Brown KK, Quintero-Rivera F, South ST. American College of Medical Genetics standards and guidelines for interpretation and reporting of postnatal constitutional copy number variants. Genet Med. 2011;13(7):680-5.

27. MacDonald JR, Ziman R, Yuen RK, Feuk L, Scherer SW. The database of genomic variants: a curated collection of structural variation in the human genome. Nucleic Acids Res. 2014:42(Database issue):D986-92.

28. Hamosh A, Scott AF, Amberger J, Valle D, McKusick VA. Online Mendelian inheritance in man (OMIM). Hum Mutat. 2000:15(1):57-61.

29. Firth HV, Richards SM, Bevan AP, Clayton S, Corpas M, Rajan D, et al. DECIPHER: database of chromosomal imbalance and phenotype in humans using Ensembl resources. Am J Hum Genet. 2009;84(4):524-33. 
30. Kent WJ, Sugnet CW, Furey TS, Roskin KM, Pringle TH, Zahler AM, Haussler D. The human genome browser at UCSC. Genome Res. 2002;12(6):996-1006.

31. Dai HJ, Wu JC, Tsai RT, Pan WH, Hsu WL. T-HOD: a literature-based candidate gene database for hypertension, database (Oxford) 2013; 2013. p. bas061.

32. Yu W, Clyne M, Khoury MJ, Gwinn M. Phenopedia and Genopedia: diseasecentered and gene-centered views of the evolving knowledge of human genetic associations. Bioinformatics. 2010;26(1):145-6.

33. Butler MG, McGuire A, Manzardo AM. Clinically relevant known and candidate genes for obesity and their overlap with human infertility and reproduction. J Assist Reprod Genet. 2015;32(4):495-508.

34. Coe BP, Witherspoon $K$, Rosenfeld JA, van Bon BW, Vulto-van Silfhout AT, Bosco P, et al. Refining analyses of copy number variation identifies specific genes associated with developmental delay. Nat Genet. 2014;46:1063-71.

35. Lopes CT, Franz M, Kazi F, Donaldson SL, Morris Q, Bader GD. Cytoscape web: an interactive web-based network browser. Bioinformatics. 2010; 15(26(18)):2347-8

36. D'Angelo CS, Kohl I, Varela MC, de Castro Cl, Kim CA, Bertola DR, et al. Obesity with associated developmental delay and/or learning disability in patients exhibiting additional features: report of novel pathogenic copy number variants. Am J Med Genet A. 2013;161A:479-86.

37. D'Angelo CS, Varela MC, de Castro Cl, Kim CA, Bertola DR, Lourenço CM, et al. Investigation of selected genomic deletions and duplications in a cohort of 338 patients presenting with syndromic obesity by multiplex ligationdependent probe amplification using synthetic probes. Mol Cytogenet. 2014;7:75.

38. D'Angelo CS, Moller dos Santos MF, Alonso LG, Koiffmann CP. Two new cases of 1p21.3 deletions and an unbalanced translocation t(8;12) among individuals with Syndromic obesity. Mol Syndromol. 2015;6(2):63-70.

39. Avila M, Kirchhoff M, Marle N, Hove HD, Chouchane M, Thauvin-Robinet C, et al. Delineation of a new chromosome 20q11.2 duplication syndrome including the ASXL1 gene. Am J Med Genet A. 2013;161A(7):1594-8.

40. Gimelli S, Capra V, Di Rocco M, Leoni M, Mirabelli-Badenier M, Schiaffino MC, et al. Interstitial 7q31.1 copy number variations disrupting IMMP2L gene are associated with a wide spectrum of neurodevelopmental disorders. Mol Cytogenet. 2014;7:54

41. Lionel AC, Tammimies K, Vaags AK, Rosenfeld JA, Ahn JW, Merico D, et al. Disruption of the ASTN2/TRIM32 locus at 9q33.1 is a risk factor in males for autism spectrum disorders, ADHD and other neurodevelopmental phenotypes. Hum Mol Genet. 2014;23(10):2752-68.

42. Vergult S, Dheedene A, Meurs A, Faes F, Isidor B, Janssens S, et al. Genomic aberrations of the CACNA2D1 gene in three patients with epilepsy and intellectual disability. Eur J Hum Genet. 2015;23(5):628-32.

43. Jones RM, Cadby G, Blangero J, Abraham LJ, Whitehouse AJ, Moses EK. MACROD2 gene associated with autistic-like traits in a general population sample. Psychiatr Genet. 2014;24(6):241-8.

44. Matsunami N, Hadley D, Hensel CH, Christensen GB, Kim C, Frackelton E, et al. Identification of rare recurrent copy number variants in high-risk autism families and their prevalence in a large ASD population. PLoS One. 2013;8: e52239

45. Rehm HL, Berg JS, Brooks LD, Bustamante CD, Evans JP, Landrum MJ, et al. ClinGen-the clinical genome resource. N Engl J Med. 2015;372:2235-42.

46. Lang B, Pu J, Hunter I, Liu M, Martin-Granados C, Reilly TJ, et al. Recurrent deletions of ULK4 in schizophrenia: a gene crucial for neuritogenesis and neuronal motility. J Cell Sci. 2014 Feb 1:127(Pt 3):630-40.

47. Neale BM, Kou Y, Liu L, Ma'ayan A, Samocha KE, Sabo A, et al. Patterns and rates of exonic de novo mutations in autism spectrum disorders. Nature. 2012 Apr 4;485(7397):242-5.

48. Bonnefond A, Raimondo A, Stutzmann F, Ghoussaini M, Ramachandrappa S, Bersten DC, et al. Loss-of-function mutations in SIM1 contribute to obesity and Prader-Willi-like features. J Clin Invest. 2013;123(7):3037-41.

49. Doche ME, Bochukova EG, Su HW, Pearce LR, Keogh JM, Henning E, et al. Human $\mathrm{SH} 2 \mathrm{~B} 1$ mutations are associated with maladaptive behaviors and obesity. J Clin Invest. 2012;122:4732-6.

50. Leroy C, Landais E, Briault S, David A, Tassy O, Gruchy N, et al. The 2q37deletion syndrome: an update of the clinical spectrum including overweight, brachydactyly and behavioural features in 14 new patients. Eur J Hum Genet. 2013;21:602-12.

51. Willemsen MH, Vulto-van Silfhout AT, Nillesen WM, Wissink-Lindhout WM, van Bokhoven H, Philip N, et al. Update on Kleefstra Syndrome. Mol Syndromol. 2012;2(3-5):202-12.
52. De Rocker N, Vergult S, Koolen D, Jacobs E, Hoischen A, Zeesman S, et al. Refinement of the critical 2p25.3 deletion region: the role of MYT1L in intellectual disability and obesity. Genet Med. 2015;17:460-6.

53. Burns B, Schmidt K, Williams SR, Kim S, Girirajan S, Elsea SH. Rai1 haploinsufficiency causes reduced Bdnf expression resulting in hyperphagia, obesity and altered fat distribution in mice and humans with no evidence of metabolic syndrome. Hum Mol Genet. 2010;19:4026-42.

54. Kogelman LJA, Zhernakova DV, Westra H-J, Cirera S, Fredholm M, Franke L, Kadarmideen HN. An integrative systems genetics approach reveals potential causal genes and pathways related to obesity. Genome Med. 2015:7:105.

55. Bassett AS, Chow EW, Husted J, Weksberg R, Caluseriu O, Webb GD, Gatzoulis MA. Clinical features of 78 adults with 22 a11 deletion syndrome. Am J Med Genet A. 2005;138(4):307-13

56. Bassett AS, McDonald-McGinn DM, Devriendt K, Digilio MC, Goldenberg P, Habel A, et al. Practical guidelines for managing patients with 22q11.2 deletion syndrome. J Pediatr. 2011:159(2):332-9.

57. Voll SL, Boot E, Butcher NJ, Cooper S, Heung T, Chow EW, et al. Obesity in adults with 22q11.2 deletion syndrome. Genet Med. 2016; https://doi.org/10. 1038/gim.2016.98.

58. D'Angelo CS, Jehee FS, Koiffmann CP. An inherited atypical 1 Mb 22q11.2 deletion within the DGSNCFS $3 \mathrm{Mb}$ region in a child with obesity and aggressive behavior. Am J Med Genet A. 2007;143A:1928-32.

59. Bassett JK, Chandler KE, Douzgou S. Two patients with chromosome 22q11. 2 deletion presenting with childhood obesity and hyperphagia. Eur J Med Genet. 2016:59(8):401-3.

60. Mikhail FM, Descartes M, Piotrowski A, Andersson R, Diaz de Ståhl T, Komorowski J, et al. A previously unrecognized microdeletion syndrome on chromosome 22 band q11.2 encompassing the BCR gene. Am J Med Genet A. 2007:43A(18):2178-84

61. Ben-Shachar S, Ou Z, Shaw CA, Belmont JW, Patel MS, Hummel M, et al. 22q11.2 distal deletion: a recurrent genomic disorder distinct from DiGeorge syndrome and velocardiofacial syndrome. Am J Hum Genet. 2008;82:214-21

62. Fagerberg CR, Graakjaer J, Heinl UD, Ousager LB, Dreyer I, Kirchhoff M, et al. Heart defects and other features of the 22q11 distal deletion syndrome. Eur J Med Genet. 2013;56(2):98-107.

63. Mikhail FM, Burnside RD, Rush B, Ibrahim J, Godshalk R, Rutledge SL, et al. The recurrent distal 22q11.2 microdeletions are often de novo and do not represent a single clinical entity: a proposed categorization system. Genet Med. 2013;16(1):92-100.

64. Hauge X, Raca G, Cooper S, May K, Spiro R, Adam M, Martin L. Detailed characterization of and clinical correlations in ten patients with distal deletions of chromosome 9p. Genet Med. 2008;10(8):599-611.

65. Pober BR. Williams-Beuren syndrome. New Eng J Med. 2010;362:239-52.

66. Stagi S, Lapi E, Cecchi C, Chiarelli F, D'Avanzo MG, Seminara S, et al. Williams-Beuren syndrome is a genetic disorder associated with impaired glucose tolerance and diabetes in childhood and adolescence. New insights from a longitudinal study. Horm Res Pediatr. 2014;82:38-43.

67. Tropeano M, Ahn JW, Dobson RJB, Breen G, Rucker J, Dixit A, et al. MaleBiased Autosomal Effect of 16p13.11 Copy Number Variation in Neurodevelopmental Disorders. PLoS One. 2013;8(4):e61365.

68. Dolcetti A, Silversides CK, Marshall CR, Lionel AC, Stavropoulos DJ, Scherer SW, Bassett AS. 1q21.1 microduplication expression in adults. Genet Med. 2013:15:282-9.

69. Bartholdi D, Stray-Pedersen A, Azzarello-Burri S, Kibaek M, Kirchhoff M, Oneda B, et al. A newly recognized 13q12.3 microdeletion syndrome characterized by intellectual disability, microcephaly, and eczema/atopic dermatitis encompassing the HMGB1 and KATNAL1 genes. Am J Med Genet A. 2014;164A(5):1277-83.

70. Kaaman M, Ryden M, Axelsson T, Nordstrom E, Sicard A, Bouloumie A, et al. ALOX5AP expression, but not gene haplotypes, is associated with obesity and insulin resistance. Int J Obes. 2006;30:447-52.

71. Bonaglia MC, Marelli S, Novara F, Commodaro S, Borgatti R, Minardo G, et al. Genotype-phenotype relationship in three cases with overlapping 19p13.12 microdeletions. Eur J Hum Genet. 2010;18(12):1302-9.

72. Bi P, Kuang S. Notch signaling as a novel regulator of metabolism. Trends Endocrinol Metab. 2015;26(5):248-55.

73. Lehman AM, du Souich C, Chai D, Eydoux P, Huang JL, Fok AK, et al. 19p13. 2 microduplication causes a Sotos syndrome-like phenotype and alters gene expression. Clin Genet. 2010;81:56-63. 
74. McManaman JL, Bales ES, Orlicky DJ, Jackman M, MacLean PS, Cain S, et al. Perilipin-2-null mice are protected against diet-induced obesity, adipose inflammation, and fatty liver disease. J Lipid Res. 2013;54(5):1346-59.

75. Wang K, Li WD, Glessner JT, Grant SF, Hakonarson H, Price RA. Large copynumber variations are enriched in cases with moderate to extreme obesity. Diabetes. 2010;59(10):2690-4.

76. Saxena R, Saleheen D, Been LF, Garavito ML, Braun T, Bjonnes A, et al. Genome-wide association study identifies a novel locus contributing to type 2 diabetes susceptibility in Sikhs of Punjabi origin from India. Diabetes. 2013;62:1746-55.

77. Wu K-S, Hung Y-J, Lee C-H, Hsiao F-C, Hsieh P-S. The involvement of GAS6 signaling in the development of obesity and associated inflammation. Int J Endocrinol. 2015:2015:202513.

78. Tassano E, Alpigiani MG, Calcagno A, Salvati P, De Miglio L, Fiorio P, Gimell G. Clinical and molecular delineation of a 16p13.2p13.13 microduplication. Eur J Med Genet. 2015;58(3):194-8.

79. Hao YH, Fountain MD Jr, Fon Tacer K, Xia F, Bi W, Kang SH, et al. USP7 acts as a molecular rheostat to promote WASH-dependent Endosomal protein recycling and is mutated in a human Neurodevelopmental disorder. Mol Cell. 2015;59(6):956-69.

Submit your next manuscript to BioMed Central and we will help you at every step:

- We accept pre-submission inquiries

- Our selector tool helps you to find the most relevant journal

- We provide round the clock customer support

- Convenient online submission

- Thorough peer review

- Inclusion in PubMed and all major indexing services

- Maximum visibility for your research

Submit your manuscript at www.biomedcentral.com/submit
Biomed Central 